\title{
Uniform Stability and Error Analysis for Some Discontinuous Galerkin Methods
}

\author{
Qingguo Hong and Jinchao Xu
}

\begin{abstract}
In this paper, we provide a number of new estimates on the stability and convergence of both hybrid discontinuous Galerkin (HDG) and weak Galerkin (WG) methods. By using the standard Brezzi theory on mixed methods, we carefully define appropriate norms for the various discretization variables and then establish that the stability and error estimates hold uniformly with respect to stabilization and discretization parameters. As a result, by taking appropriate limit of the stabilization parameters, we show that the HDG method converges to a primal conforming method and the WG method converge to a mixed conforming method.
\end{abstract}

Keywords. Uniform Stability, Uniform Error Estimate, Hybrid Discontinuous Galerkin, Weak Galerkin

\section{Introduction}

In the last few decades, one variant of finite element method called the discontinuous Galerkin (DG) method $[1,2]$ has been developed to solve various differential equations due to their flexibility in constructing feasible local shape-function spaces and the advantage of effectively capturing non-smooth or oscillatory solutions. Since DG methods use discontinuous space as trial space, the number of degrees of freedom is usually much higher than the standard conforming method. To reduce the number of globally coupled degrees of freedom of DG methods, a hybrid DG (HDG) has been developed. The idea of hybrid methods can be tracked to the 1960s [3]. A new hybridization approach in [4] was put forward by Cockburn and Gopalakrishnan in 2004 and was successfully applied to a discontinuous Galerkin method in [5]. Using the local discontinuous Galerkin (LDG) method to define the local solvers, a super-convergent LDG-hybridizable Galerkin method for second-order elliptic problems was designed in [6]. In 2009, a unified analysis for the hybridization of discontinuous Galerkin, mixed, and continuous Galerkin methods for second-order elliptic problems was presented in [7] by Cockburn, Gopalakrishnan, and Lazarov. A projection-based error analysis of HDG 
methods was presented in [8], where a projection was constructed to obtain the $L^{2}$ error estimate for the potential and flux. However, the error estimate was dependent on the stabilization parameter. A projectionbased analysis of the hybridized discontinuous Galerkin methods for convection-diffusion equations for semimatching nonconforming meshes was presented in [9]. An analysis for a hybridized discontinuous Galerkin method with reduced stabilization for second-order elliptic problem was given in [10].

Based on a new concept, namely the weak gradient, introduced in [11], Wang and Ye proposed a weak Galerkin (WG) method for elliptic equations. Similar to the concept introduced in [11], Wang and Ye [12] introduced a concept called weak divergence. Based on the newly introduced concept, Wang and Ye [12] proposed and analyzed a WG method for the second-order elliptic equation formulated as a system of two first-order linear equations. Then a similar idea was applied to Darcy-Stokes flow in [13]. A primal-dual WG finite element method for second-order elliptic equations in non-divergence form was presented in [14] and a further similar method was applied to Fokker-Planck type equations in [15]. A bridge building the connection between the WG method and HDG method was shown in [16]. A summary of the idea and applications of WG methods to various problem were provided in [17].

In this paper, in contrast to the projection-based error analysis in $[8,10]$, we use the LadyzhenskayaBabuška-Brezzi (LBB) theory to prove two types of uniform stability results under some carefully constructed parameter-dependent norms for HDG methods. Based on the uniform stability results, we prove uniform and optimal error estimates for HDG methods. In addition, by using properly defined parameter-dependent norms, we further prove two types of uniform stability results for WG methods. Similarly based on the uniform stability results, we provide uniform and optimal error estimates for WG methods. These uniform stability results and error estimates for WG methods are meaningful and interesting improvement for the results in $[11,12]$. Following these uniform stability results for HDG methods and WG methods presented in this paper, an HDG method is shown to converge to a primal conforming method, whereas a WG method is shown to converge to a mixed conforming method by taking the limit of the stabilization parameters.

We illustrate the main idea and results by using the following elliptic boundary value problem:

$$
\left\{\begin{aligned}
-\operatorname{div}(\alpha \nabla u)=f & \text { in } \Omega, \\
u=0 & \text { on } \partial \Omega,
\end{aligned}\right.
$$

where $\Omega \subset \mathbb{R}^{d}(d \geq 1)$ is a bounded domain and $\alpha: \mathbb{R}^{d} \rightarrow \mathbb{R}^{d}$ is a bounded and symmetric positive definite matrix, and its inverse is denoted by $c=\alpha^{-1}$. Setting $\boldsymbol{p}=-\alpha \nabla u$, the above problem can be written as:

$$
\left\{\begin{array}{rc}
c \boldsymbol{p}+\nabla u=0 & \text { in } \Omega, \\
-\operatorname{div} \boldsymbol{p}=f & \text { in } \Omega, \\
u=0 & \text { on } \partial \Omega .
\end{array}\right.
$$


The rest of the paper is organized as follows. In Section 2, some preliminary materials are provided. In Section 3, we set up the HDG and WG methods and provide the main uniform well-posedness results. Based on the uniform well-posedness results, we present uniform and optimal error estimates for HDG and WG in Section 4, and show that an HDG method converges to a primal conforming method, whereas a WG method converges to a mixed conforming method by taking the limit of the stabilization parameters in Section 5. In Section 6, we provide proof of the uniform well-posedness of HDG and WG under the specific parameter-dependent norms. We provide a brief summary in the last section.

\section{Preliminaries}

In this section, we describe some basic notation. Throughout this paper, we use letter $C$ to denote a generic positive constant, which may stand for different values at different occurrences, but not depending the mesh size and the stability parameters. The notations $x \lesssim y$ and $x \gtrsim y$ mean $x \leq C y$ and $x \geq C y$, respectively.

\subsection{Discontinuous Galerkin Notation}

Given a bounded domain $D \subset \mathbb{R}^{d}$ and a positive integer $m, H^{m}(D)$ is the Sobolev space with the corresponding usual norm and semi-norm, which are denoted respectively by $\|\cdot\|_{m, D}$ and $|\cdot|_{m, D}$. We abbreviate them by $\|\cdot\|_{m}$ and $|\cdot|_{m}$, respectively, when $D$ is chosen as $\Omega$. The $L^{2}$-inner products on $D$ and $\partial D$ are denoted by $(\cdot, \cdot)_{D}$ and $\langle\cdot, \cdot\rangle_{\partial D}$, respectively. Moreover, $\|\cdot\|_{0, D}$ and $\|\cdot\|_{0, \partial D}$ are the norms of Lebesgue spaces $L^{2}(D)$ and $L^{2}(\partial D)$, respectively, and $\|\cdot\|=\|\cdot\|_{0, \Omega}$. We also set $H(\operatorname{div}, \Omega)=\left\{\boldsymbol{u} \in \boldsymbol{L}^{\mathbf{2}}(\boldsymbol{\Omega}): \operatorname{div} \boldsymbol{u} \in L^{2}(\Omega)\right\}$ equipped with the norm $\|\boldsymbol{u}\|_{\operatorname{div}}^{2}=(\boldsymbol{u}, \boldsymbol{u})+(\operatorname{div} \boldsymbol{u}, \operatorname{div} \boldsymbol{u})$.

We assume $\Omega$ is a polygonal domain, and a family of triangulations of $\bar{\Omega}$ is denoted by $\left\{\mathcal{T}_{h}\right\}_{h}$, with the minimal angle condition satisfied. Let $h_{K}=\operatorname{diam}(K)$ and $h=\max \left\{h_{K}: K \in \mathcal{T}_{h}\right\}$. We denote $\mathcal{E}_{h}^{i}$ the set of interior edges (or faces) of $\mathcal{T}_{h}$ and $\mathcal{E}_{h}^{\partial}$ the set of boundary edges (or faces), and let $\mathcal{E}_{h}=\mathcal{E}_{h}^{\partial} \cup \mathcal{E}_{h}^{i}$. For $e \in \mathcal{E}_{h}$, let $h_{e}=\operatorname{diam}(e)$. For $e \in \mathcal{E}_{h}^{i}$, we choose a fixed normal unit direction denoted by $\mathbf{n}_{e}$, and for $e \in \mathcal{E}_{h}^{\partial}$, we take the outward unit normal as $\mathbf{n}_{e}$. Let $e$ be the common edge of two elements $K^{+}$and $K^{-}$, and $\boldsymbol{n}^{i}=\left.\boldsymbol{n}\right|_{\partial K^{i}}$ be the unit outward normal vector on $\partial K^{i}$ with $i=+,-$. For any scalar-valued function $v$ and vector-valued function $\boldsymbol{q}$, let $v^{ \pm}=\left.v\right|_{\partial K^{ \pm}}$and $\boldsymbol{q}^{ \pm}=\left.\boldsymbol{q}\right|_{\partial K^{ \pm}}$. Then, we define averages $\{\cdot\},\{[\cdot\}$ and jumps $\llbracket \cdot \rrbracket,[\cdot]$ as follows:

$$
\begin{array}{llrl}
\{v\}=\frac{1}{2}\left(v^{+}+v^{-}\right), \quad\{\boldsymbol{q}\}=\frac{1}{2}\left(\boldsymbol{q}^{+}+\boldsymbol{q}^{-}\right), \quad\{q \boldsymbol{q}\}=\frac{1}{2}\left(\boldsymbol{q}^{+} \cdot \boldsymbol{n}^{+}-\boldsymbol{q}^{-} \cdot \boldsymbol{n}^{-}\right) & & \text {on } e \in \mathcal{E}_{h}^{i}, \\
\llbracket v \rrbracket=v^{+} \boldsymbol{n}^{+}+v^{-} \boldsymbol{n}^{-}, & {[v]=v^{+}-v^{-}, \quad[\boldsymbol{q}]=\boldsymbol{q}^{+} \cdot \boldsymbol{n}^{+}+\boldsymbol{q}^{-} \cdot \boldsymbol{n}^{-}} & & \text {on } e \in \mathcal{E}_{h}^{i}, \\
\llbracket v \rrbracket=v \boldsymbol{n}, \quad[v]=v, & \{\boldsymbol{q}\}=\boldsymbol{q}, \quad\{\boldsymbol{q}\}=\boldsymbol{q} \cdot \boldsymbol{n} & & \text { on } e \in \mathcal{E}_{h}^{\partial} .
\end{array}
$$

Here, we specify $\boldsymbol{n}$ as the outward unit normal direction on $\partial \Omega$. 
We define some inner products as follows:

$$
(\cdot, \cdot)_{\mathcal{T}_{h}}=\sum_{K \in \mathcal{T}_{h}}(\cdot, \cdot)_{K}, \quad\langle\cdot, \cdot\rangle_{\mathcal{E}_{h}}=\sum_{e \in \mathcal{E}_{h}}\langle\cdot, \cdot\rangle_{e}, \quad\langle\cdot, \cdot\rangle_{\mathcal{E}_{h}^{i}}=\sum_{e \in \mathcal{E}_{h}^{i}}\langle\cdot, \cdot\rangle_{e}, \quad\langle\cdot, \cdot\rangle_{\partial \mathcal{T}_{h}}=\sum_{K \in \mathcal{T}_{h}}\langle\cdot, \cdot\rangle_{\partial K} .
$$

We now give more details about the last notation of the inner product. For any scalar-valued function $v$ and vector-valued function $\boldsymbol{q}$,

$$
\langle v, \boldsymbol{q} \cdot \boldsymbol{n}\rangle_{\partial \mathcal{T}_{h}}=\sum_{K \in \mathcal{T}_{h}}\langle v, \boldsymbol{q} \cdot \boldsymbol{n}\rangle_{\partial K}=\sum_{K \in \mathcal{T}_{h}}\left\langle v, \boldsymbol{q} \cdot \boldsymbol{n}_{K}\right\rangle_{\partial K} \cdot
$$

Here, we specify the outward unit normal direction $\boldsymbol{n}$ corresponding to the element $K$, namely $\boldsymbol{n}_{K}$.

For the piecewise smooth scalar-valued function $v$ and vector-valued function $\boldsymbol{q}$, let $\nabla_{h}$ and $\operatorname{div}_{h}$ be defined by the relation

$$
\left.\left(\nabla_{h} v\right)\right|_{K}=\nabla\left(\left.v\right|_{K}\right),\left.\quad\left(\operatorname{div}_{h} \boldsymbol{q}\right)\right|_{K}=\operatorname{div}\left(\left.\boldsymbol{q}\right|_{K}\right),
$$

on any element $K \in \mathcal{T}_{h}$, respectively.

With the definition of averages and jumps, we have the following identity:

$$
\langle v, \boldsymbol{q} \cdot \boldsymbol{n}\rangle_{\partial T_{h}}=\langle\{\boldsymbol{q}\}, \llbracket v \rrbracket\rangle_{\mathcal{E}_{h}}+\langle[\boldsymbol{q}],\{v\}\rangle_{\mathcal{E}_{h}^{i}},
$$

and

$$
\{\boldsymbol{q}\} \cdot \llbracket v \rrbracket=\{\{\boldsymbol{q}\} \cdot[v] .
$$

Before discussing various Galerkin methods, we need to introduce the finite element spaces associated with the triangulation $\mathcal{T}_{h}$. First, $V_{h}$ and $\boldsymbol{Q}_{h}$ are the piecewise scalar and vector-valued discrete spaces on the triangulation $\mathcal{T}_{h}$, respectively and for $k \geq 0$, we define the spaces as follows:

$$
\begin{aligned}
V_{h}^{k} & =\left\{v_{h} \in L^{2}(\Omega):\left.v_{h}\right|_{K} \in \mathcal{P}_{k}(K), \forall K \in \mathcal{T}_{h}\right\}, \\
\boldsymbol{Q}_{h}^{k} & =\left\{\boldsymbol{p}_{h} \in \boldsymbol{L}^{2}(\boldsymbol{\Omega}):\left.\boldsymbol{p}_{h}\right|_{K} \in \mathcal{P}_{\boldsymbol{k}}(\boldsymbol{K}), \forall K \in \mathcal{T}_{h}\right\}, \\
\boldsymbol{Q}_{h}^{k, R T} & =\left\{\boldsymbol{p}_{h} \in \boldsymbol{L}^{\mathbf{2}}(\boldsymbol{\Omega}):\left.\boldsymbol{p}_{h}\right|_{K} \in \mathcal{P}_{\boldsymbol{k}}(\boldsymbol{K})+\boldsymbol{x} P_{k}(K), \forall K \in \mathcal{T}_{h}\right\},
\end{aligned}
$$

where $\mathcal{P}_{k}(K)$ is the space of polynomial functions of degree at most $k$ on $K$. We also use the following spaces associated with $\mathcal{E}_{h}$ :

$$
\begin{aligned}
& \hat{\boldsymbol{Q}}_{h}=\left\{\hat{\boldsymbol{p}}_{h}:\left.\hat{\boldsymbol{p}}_{h}\right|_{e} \in \hat{Q}(e) \boldsymbol{n}_{e}, \forall e \in \mathcal{E}_{h}\right\}, \\
& \hat{Q}_{h}=\left\{\hat{p}_{h}:\left.\hat{p}_{h}\right|_{e} \in \hat{Q}(e), \forall e \in \mathcal{E}_{h}\right\}, \\
& \hat{V}_{h}=\left\{\hat{v}_{h}:\left.\hat{v}_{h}\right|_{e} \in \hat{V}(e), e \in \mathcal{E}_{h}^{i},\left.\hat{v}_{h}\right|_{\mathcal{E}_{h}^{\partial}}=0\right\}, \\
& \hat{Q}_{h}^{k}=\left\{\hat{p}_{h} \in L^{2}\left(\mathcal{E}_{h}\right):\left.\hat{p}_{h}\right|_{e} \in \mathcal{P}_{k}(e), \forall e \in \mathcal{E}_{h}\right\}, \\
& \hat{V}_{h}^{k}=\left\{\hat{v}_{h} \in L^{2}\left(\mathcal{E}_{h}\right):\left.\hat{v}_{h}\right|_{e} \in \mathcal{P}_{k}(e), \forall e \in \mathcal{E}_{h}^{i},\left.\hat{v}_{h}\right|_{\mathcal{E}_{h}^{\partial}}=0\right\},
\end{aligned}
$$

where $\hat{Q}(e)$ and $\hat{V}(e)$ are some local spaces on $e$ and $\mathcal{P}_{k}(e)$ is the space of polynomial functions of degree at most $k$ on $e$. For convenience, we denote $\tilde{\boldsymbol{Q}}_{h}=\boldsymbol{Q}_{h} \times \hat{\boldsymbol{Q}}_{h}$ and $\tilde{V}_{h}=V_{h} \times \hat{V}_{h}$. 


\section{Uniform Stability for HDG and WG Methods}

In this section, we set up the HDG and WG methods first and then provide the uniform well-posedness for both HDG and WG methods under proper parameter-dependent defined norms.

\subsection{Setting up the HDG and WG Methods}

Now we start with the second-order elliptic equation and set $\boldsymbol{p}=-\alpha \nabla u$ to obtain the following form:

$$
\left\{\begin{aligned}
c \boldsymbol{p}+\nabla u=0 & \text { in } \Omega, \\
\operatorname{div} \boldsymbol{p}=f & \text { in } \Omega .
\end{aligned}\right.
$$

Multiplying the first and second equations by $\boldsymbol{q}_{h} \in \boldsymbol{Q}_{h}$ and $v_{h} \in V_{h}$, respectively, then integrating on an element $K \in \mathcal{T}_{h}$, we obtain:

$$
\left\{\begin{array}{cl}
\left(c \boldsymbol{p}, \boldsymbol{q}_{h}\right)_{K}-\left(u, \operatorname{div} \boldsymbol{q}_{h}\right)_{K}+\left\langle u, \boldsymbol{q}_{h} \cdot \boldsymbol{n}_{K}\right\rangle_{\partial K}=0 & \forall \boldsymbol{q}_{h} \in \boldsymbol{Q}_{h}, \\
\left(\boldsymbol{p}, \nabla v_{h}\right)_{K}-\left\langle\boldsymbol{p} \cdot \boldsymbol{n}_{K}, v_{h}\right\rangle_{\partial K}=-\left(f, v_{h}\right)_{K} & \forall v_{h} \in V_{h} .
\end{array}\right.
$$

Summing on all $K \in \mathcal{T}_{h}$, we have:

$$
\left\{\begin{array}{l}
\left(c \boldsymbol{p}, \boldsymbol{q}_{h}\right)_{\mathcal{T}_{h}}-\left(u, \operatorname{div}_{h} \boldsymbol{q}_{h}\right)_{\mathcal{T}_{h}}+\left\langle u, \boldsymbol{q}_{h} \cdot \boldsymbol{n}\right\rangle_{\partial \mathcal{T}_{h}}=0 \quad \forall \boldsymbol{q}_{h} \in \boldsymbol{Q}_{h}, \\
\left(\boldsymbol{p}, \nabla_{h} v_{h}\right)_{\mathcal{T}_{h}}-\left\langle\boldsymbol{p} \cdot \boldsymbol{n}, v_{h}\right\rangle_{\partial \mathcal{T}_{h}}=-\left(f, v_{h}\right)_{\mathcal{T}_{h}} \quad \forall v_{h} \in V_{h} .
\end{array}\right.
$$

Now we approximate $u, \boldsymbol{p}$ by $u_{h} \in V_{h}$, and $\boldsymbol{p}_{h} \in \boldsymbol{Q}_{h}$, respectively, and the trace of $u$ and the flux $\boldsymbol{p} \cdot \boldsymbol{n}$ on $\partial K$ by $\check{u}_{h}, \check{\boldsymbol{p}}_{h} \cdot \boldsymbol{n}$. Hence, we have:

$$
\left\{\begin{array}{l}
\left(c \boldsymbol{p}_{h}, \boldsymbol{q}_{h}\right)_{\mathcal{T}_{h}}-\left(u_{h}, \operatorname{div}_{h} \boldsymbol{q}_{h}\right)_{\mathcal{T}_{h}}+\left\langle\check{u}_{h}, \boldsymbol{q}_{h} \cdot \boldsymbol{n}\right\rangle_{\partial \mathcal{T}_{h}}=0, \forall \boldsymbol{q}_{h} \in \boldsymbol{Q}_{h}, \\
\left(\boldsymbol{p}_{h}, \nabla_{h} v_{h}\right)_{\mathcal{T}_{h}}-\left\langle\check{\boldsymbol{p}}_{h} \cdot \boldsymbol{n}, v_{h}\right\rangle_{\partial \mathcal{T}_{h}}=-\left(f, v_{h}\right)_{\mathcal{T}_{h}} \quad \forall v_{h} \in V_{h} .
\end{array}\right.
$$

Next, we need to derive appropriate equations for the variables of $\check{u}_{h}$ and $\check{\boldsymbol{p}}_{h}$. The starting point is the following relationship:

$$
\check{\boldsymbol{p}}_{h} \cdot \boldsymbol{n}_{K}+\tau \check{u}_{h}=\boldsymbol{p}_{h} \cdot \boldsymbol{n}_{K}+\tau u_{h}, \quad \check{\boldsymbol{p}}_{h}=\check{p}_{h} \boldsymbol{n}_{e}
$$

The idea is that we only use either $\check{p}_{h}$ or $\check{u}_{h}$ as an unknown and then use (3.5) to determine the other variable. There are two different approaches; one approach is for deriving HDG methods, and the other one is for deriving WG methods.

First approach: (Hybridized Discontinuous Galerkin) Set $\check{u}_{h}=\hat{u}_{h} \in \hat{V}_{h}$ as an unknown that is single-valued. The "continuity" of $\check{\boldsymbol{p}}_{h}$ is then enforced weakly as follows:

$$
\left\langle\check{\boldsymbol{p}}_{h} \cdot \boldsymbol{n}, \hat{v}_{h}\right\rangle_{\partial \mathcal{T}_{h}}=0, \forall \hat{v}_{h} \in \hat{V}_{h} .
$$


where $\check{\boldsymbol{p}}_{h}$ is given by (3.5). From the identity $(2.2)$ and the fact that $\left[\hat{v}_{h}\right]=0$, a straightforward calculation shows that (3.6) can be rewritten as:

$$
\left\langle\left[\check{p}_{h}\right], \hat{v}_{h}\right\rangle_{\mathcal{E}_{h}}:=\sum_{e \in \mathcal{E}_{h}}\left\langle\left[\check{p}_{h}\right], \hat{v}_{h}\right\rangle_{e}=0 \quad \forall \hat{v}_{h} \in \hat{V}_{h}
$$

Collecting (3.4), (3.5), and (3.6), the HDG methods read: Find $\left(\boldsymbol{p}_{h}, \tilde{u}_{h}\right) \in \boldsymbol{Q}_{h} \times \tilde{V}_{h}$ such that for any $\left(\boldsymbol{q}_{h}, \tilde{v}_{h}\right) \in \boldsymbol{Q}_{h} \times \tilde{V}_{h}$,

$$
\left\{\begin{array}{l}
a_{h}\left(\boldsymbol{p}_{h}, \boldsymbol{q}_{h}\right)+b_{h}\left(\boldsymbol{q}_{h}, \tilde{u}_{h}\right)=0, \\
b_{h}\left(\boldsymbol{p}_{h}, \tilde{v}_{h}\right)+c_{h}\left(\tilde{u}_{h}, \tilde{v}_{h}\right)=-\left(f, v_{h}\right)_{\mathcal{T}_{h}} .
\end{array}\right.
$$

Here

$$
\left\{\begin{array}{l}
a_{h}\left(\boldsymbol{p}_{h}, \boldsymbol{q}_{h}\right)=\left(c \boldsymbol{p}_{h}, \boldsymbol{q}_{h}\right)_{\mathcal{T}_{h}}, \\
b_{h}\left(\boldsymbol{q}_{h}, \tilde{u}_{h}\right)=-\left(u_{h}, \operatorname{div} \boldsymbol{q}_{h}\right)_{\mathcal{T}_{h}}+\left\langle\hat{u}_{h}, \boldsymbol{q}_{h} \cdot \boldsymbol{n}_{K}\right\rangle_{\partial \mathcal{T}_{h}}, \\
c_{h}\left(\tilde{u}_{h}, \tilde{v}_{h}\right)=-\tau\left\langle u_{h}-\hat{u}_{h}, v_{h}-\hat{v}_{h}\right\rangle_{\partial \mathcal{T}_{h}}
\end{array}\right.
$$

where $\tau$ is the stabilization parameter.

The HDG method can be written in a compact form: Find $\left(\boldsymbol{p}_{h}, \tilde{u}_{h}\right) \in \boldsymbol{Q}_{h} \times \tilde{V}_{h}$ such that for any $\left(\boldsymbol{q}_{h}, \tilde{v}_{h}\right) \in \boldsymbol{Q}_{h} \times \tilde{V}_{h}$,

$$
A_{h}\left(\left(\boldsymbol{p}_{h}, \tilde{u}_{h}\right),\left(\boldsymbol{q}_{h}, \tilde{v}_{h}\right)\right)=-\left(f, v_{h}\right)_{\mathcal{T}_{h}},
$$

where

$$
A_{h}\left(\left(\boldsymbol{p}_{h}, \tilde{u}_{h}\right),\left(\boldsymbol{q}_{h}, \tilde{v}_{h}\right)\right)=a_{h}\left(\boldsymbol{p}_{h}, \boldsymbol{q}_{h}\right)+b_{h}\left(\boldsymbol{q}_{h}, \tilde{u}_{h}\right)+b_{h}\left(\boldsymbol{p}_{h}, \tilde{v}_{h}\right)+c_{h}\left(\tilde{u}_{h}, \tilde{v}_{h}\right) .
$$

In the first case, we choose $\tau=\rho h_{K}$ in (3.9) and for any $\tilde{v} \in \tilde{V}_{h}$ and $\boldsymbol{q}_{h} \in \boldsymbol{Q}_{h}$, we define

$$
\left\{\begin{array}{l}
\left\|\tilde{v}_{h}\right\|_{0, \rho, h}^{2}=\left(v_{h}, v_{h}\right)_{\mathcal{T}_{h}}+\rho \sum_{e \in \mathcal{E}_{h}^{i}} h_{e}\left\langle\hat{v}_{h}, \hat{v}_{h}\right\rangle_{e} \\
\left\|\boldsymbol{q}_{h}\right\|_{\operatorname{div}, \rho, h}^{2}=\left(c \boldsymbol{q}_{h}, \boldsymbol{q}_{h}\right)_{\mathcal{T}_{h}}+\left(\operatorname{div} \boldsymbol{q}_{h}, \operatorname{div} \boldsymbol{q}_{h}\right)_{\mathcal{T}_{h}}+\rho^{-1} \sum_{e \in \mathcal{E}_{h}^{i}} h_{e}^{-1}\left\langle\hat{P}_{e}\left(\left[\boldsymbol{q}_{h}\right]\right), \hat{P}_{e}\left(\left[\boldsymbol{q}_{h}\right]\right)\right\rangle_{e}
\end{array}\right.
$$

where $\hat{P}_{e}: L^{2}(e) \rightarrow \hat{V}(e)$ is the $L^{2}$ projection.

In the second case, we choose $\tau=\rho^{-1} h_{K}^{-1}$ in (3.9) and for any $\tilde{v} \in \tilde{V}_{h}$ and $\boldsymbol{q}_{h} \in \boldsymbol{Q}_{h}$, we define

$$
\left\|\tilde{v}_{h}\right\|_{\tilde{1}, \rho, h}^{2}=\left(\nabla_{h} v_{h}, \nabla_{h} v_{h}\right)_{\mathcal{T}_{h}}+\rho^{-1} \sum_{K \in \mathcal{T}_{h}} h_{K}^{-1}\left\langle v_{h}-\hat{v}_{h}, v_{h}-\hat{v}_{h}\right\rangle_{\partial K}, \quad\left\|\boldsymbol{q}_{h}\right\|^{2}=\left(c \boldsymbol{q}_{h}, \boldsymbol{q}_{h}\right)_{\mathcal{T}_{h}}
$$

By noting that

$$
\left\langle v_{h}-\hat{v}_{h}, v_{h}-\hat{v}_{h}\right\rangle_{\partial \mathcal{T}_{h}}=2\left\langle\left\{v_{h}-\hat{v}_{h}\right\},\left\{v_{h}-\hat{v}_{h}\right\}\right\rangle_{\mathcal{E}_{h}}+\frac{1}{2}\left\langle\llbracket v_{h}-\hat{v}_{h} \rrbracket, \llbracket v_{h}-\hat{v}_{h} \rrbracket\right\rangle_{\mathcal{E}_{h}},
$$

$\left\|\tilde{v}_{h}\right\|_{\tilde{1}, \rho, h}$ is indeed a norm on $\tilde{V}_{h}$. 
Second approach: (Weak Galerkin) We set $\check{\boldsymbol{p}}_{h}:=\hat{\boldsymbol{p}}_{h}=\hat{p}_{h} \boldsymbol{n}_{e} \in \hat{\boldsymbol{Q}}_{h}$ as an unknown that is singlevalued. The "continuity" of $\check{u}_{h}$ is then enforced weakly as follows:

$$
\left\langle\check{u}_{h}, \hat{\boldsymbol{q}}_{h} \cdot \boldsymbol{n}\right\rangle_{\partial \mathcal{T}_{h}}=0 \quad \forall \hat{\boldsymbol{q}}_{h} \in \hat{\boldsymbol{Q}}_{h},
$$

where $\check{u}_{h}$ is again given by (3.5). From the identity (2.2) and the fact that $\left[\hat{\boldsymbol{q}}_{h}\right]=0$, a straightforward calculation shows that (3.14) can be rewritten as:

$$
\left\langle\left[\check{u}_{h}\right], \hat{q}_{h}\right\rangle_{\mathcal{E}_{h}}:=\sum_{e \in \mathcal{E}_{h}}\left\langle\left[\check{u}_{h}\right], \hat{q}_{h}\right\rangle_{e}=0 \quad \forall \hat{q}_{h} \in \hat{Q}_{h} .
$$

Collecting (3.4), (3.5), and (3.14), the WG methods read: Find $\left(\tilde{\boldsymbol{p}}_{h}, u_{h}\right) \in \tilde{\boldsymbol{Q}}_{h} \times V_{h}$ such that for any $\left(\tilde{\boldsymbol{q}}_{h}, v_{h}\right) \in \tilde{\boldsymbol{Q}}_{h} \times V_{h}$,

$$
\left\{\begin{array}{l}
a_{w}\left(\tilde{\boldsymbol{p}}_{h}, \tilde{\boldsymbol{q}}_{h}\right)+b_{w}\left(u_{h}, \tilde{\boldsymbol{q}}_{h}\right)=0, \\
b_{w}\left(\tilde{\boldsymbol{p}}_{h}, v_{h}\right)=-\left(f, v_{h}\right) .
\end{array}\right.
$$

Here

$$
\left\{\begin{array}{l}
a_{w}\left(\tilde{\boldsymbol{p}}_{h}, \tilde{\boldsymbol{q}}_{h}\right)=\left(c \boldsymbol{p}_{h}, \boldsymbol{q}_{h}\right)_{\mathcal{T}_{h}}+\eta\left\langle\left(\boldsymbol{p}_{h}-\hat{\boldsymbol{p}}_{h}\right) \cdot \boldsymbol{n},\left(\boldsymbol{q}_{h}-\hat{\boldsymbol{q}}_{h}\right) \cdot \boldsymbol{n}\right\rangle_{\partial \mathcal{T}_{h}}, \\
b_{w}\left(\tilde{\boldsymbol{p}}_{h}, v_{h}\right)=\left(\boldsymbol{p}_{h}, \nabla_{h} v_{h}\right)_{\mathcal{T}_{h}}-\left(\hat{\boldsymbol{p}}_{h} \cdot \boldsymbol{n}_{K}, v_{h}\right)_{\partial \mathcal{T}_{h}},
\end{array}\right.
$$

where $\eta$ is the stabilized parameter.

The WG method can be rewritten in a compact form: Find $\left(\tilde{\boldsymbol{p}}_{h}, u_{h}\right) \in \tilde{\boldsymbol{Q}}_{h} \times V_{h}$ such that for any $\left(\tilde{\boldsymbol{q}}_{h}, v_{h}\right) \in \tilde{\boldsymbol{Q}}_{h} \times V_{h}:$

$$
A_{w}\left(\left(\tilde{\boldsymbol{p}}_{h}, u_{h}\right),\left(\tilde{\boldsymbol{q}}_{h}, v_{h}\right)\right)=-\left(f, v_{h}\right),
$$

where

$$
A_{w}\left(\left(\tilde{\boldsymbol{p}}_{h}, u_{h}\right),\left(\tilde{\boldsymbol{q}}_{h}, v_{h}\right)\right)=a_{w}\left(\tilde{\boldsymbol{p}}_{h}, \tilde{\boldsymbol{q}}_{h}\right)+b_{w}\left(\tilde{\boldsymbol{q}}_{h}, u_{h}\right)+b_{w}\left(\tilde{\boldsymbol{p}}_{h}, v_{h}\right) .
$$

In the first case, we choose parameter $\eta$ as $\eta=\rho h_{K}$ in (3.17) and for any $v_{h} \in V_{h}$ and $\tilde{\boldsymbol{q}}_{h} \in \tilde{\boldsymbol{Q}}_{h}$, we define the norms as follows:

$$
\left\{\begin{array}{l}
\left\|v_{h}\right\|_{1, h, \rho}^{2}=\left\|\nabla_{h} v_{h}\right\|^{2}+\rho^{-1} \sum_{e \in \mathcal{E}_{h}} h_{e}^{-1}\left\|\hat{Q}_{e}\left(\left[v_{h}\right]\right)\right\|_{0, e}^{2}, \\
\left\|\tilde{\boldsymbol{q}}_{h}\right\|_{0, h, \rho}^{2}=\left(c \boldsymbol{q}_{h}, \boldsymbol{q}_{h}\right)_{\mathcal{T}_{h}}+\rho \sum_{K \in \mathcal{T}_{h}} h_{K}\left\langle\left(\boldsymbol{q}_{h}-\hat{\boldsymbol{q}}_{h}\right) \cdot \boldsymbol{n}_{K},\left(\boldsymbol{q}_{h}-\hat{\boldsymbol{q}}_{h}\right) \cdot \boldsymbol{n}_{K}\right\rangle_{\partial K} .
\end{array}\right.
$$

where $\hat{Q}_{e}$ is the $L^{2}$ projection from $L^{2}(e)$ to $\hat{Q}(e)$.

In the second case, we choose parameter $\eta$ as $\eta=\rho^{-1} h_{K}^{-1}$ in (3.17) and for any $v_{h} \in V_{h}$ and $\tilde{\boldsymbol{q}}_{h} \in \tilde{\boldsymbol{Q}}_{h}$, we define the norms as follows:

$$
\left\|u_{h}\right\|^{2}=\left(u_{h}, u_{h}\right)_{\mathcal{T}_{h}} ; \quad\left\|\tilde{\boldsymbol{q}}_{h}\right\|_{\widetilde{\mathrm{div}}, \rho, h}^{2}=\sum_{K \in \mathcal{T}_{h}}\left\|\tilde{\boldsymbol{q}}_{h}\right\|_{\widetilde{\mathrm{div}}, \rho, K}^{2},
$$

where $\left\|\tilde{\boldsymbol{q}}_{h}\right\|_{\widetilde{\mathrm{div}}, \rho, K}^{2}=\left(c \boldsymbol{q}_{h}, \boldsymbol{q}_{h}\right)_{K}+\left(\operatorname{div} \boldsymbol{q}_{h}, \operatorname{div} \boldsymbol{q}_{h}\right)_{K}+\rho^{-1} h_{K}^{-1}\left\langle\left(\boldsymbol{q}_{h}-\hat{\boldsymbol{q}}_{h}\right) \cdot \boldsymbol{n}_{K},\left(\boldsymbol{q}_{h}-\hat{\boldsymbol{q}}_{h}\right) \cdot \boldsymbol{n}_{K}\right\rangle_{\partial K}$. 


\subsection{Uniform Well-posedness of HDG and WG}

For the elliptic problem (1.2), we set a discretization: Find $\mathcal{U}_{\boldsymbol{h}} \in \boldsymbol{U}_{\boldsymbol{h}}$, such that:

$$
A_{h, \theta}\left(\mathcal{U}_{\boldsymbol{h}}, \mathcal{V}_{\boldsymbol{h}}\right)=F\left(\mathcal{V}_{\boldsymbol{h}}\right) \quad \forall \mathcal{V}_{\boldsymbol{h}} \in \boldsymbol{U}_{\boldsymbol{h}}
$$

where $\boldsymbol{U}_{\boldsymbol{h}}$ is a finite dimensional space according to partition $\mathcal{T}_{h}$ and $A_{h, \theta}\left(\mathcal{U}_{\boldsymbol{h}}, \mathcal{V}_{\boldsymbol{h}}\right)$ is a general symmetric $\theta$-parameter-dependent bilinear form and $F\left(\mathcal{V}_{\boldsymbol{h}}\right)=-\left(f, v_{h}\right)_{\mathcal{T}_{h}}$.

Let $\mathcal{U}=(\boldsymbol{p}, u)$ be the true solution of (1.2).

1. We say that the discretization (3.22) is consistent if

$$
A_{h, \theta}\left(\mathcal{U}, \mathcal{V}_{\boldsymbol{h}}\right)=F\left(\mathcal{V}_{\boldsymbol{h}}\right) \quad \forall \mathcal{V}_{\boldsymbol{h}} \in \boldsymbol{U}_{\boldsymbol{h}}
$$

2. We say that the bilinear form $A_{h, \theta}\left(\mathcal{U}_{\boldsymbol{h}}, \mathcal{V}_{\boldsymbol{h}}\right)$ is uniformly continuous with respect to the norm $\|\cdot\|_{\boldsymbol{U}_{h, \theta}}$ if

$$
\left|A_{h, \theta}\left(\mathcal{U}_{\boldsymbol{h}}, \mathcal{V}_{\boldsymbol{h}}\right)\right| \leq M_{0}\left\|\mathcal{U}_{\boldsymbol{h}}\right\|_{\boldsymbol{U}_{h, \theta}}\left\|\mathcal{V}_{\boldsymbol{h}}\right\|_{\boldsymbol{U}_{h, \theta}}
$$

where $M_{0}$ is independent of the parameter $\theta$ and the mesh size $h$.

3. We say that the bilinear form $A_{h, \theta}\left(\mathcal{U}_{\boldsymbol{h}}, \mathcal{V}_{\boldsymbol{h}}\right)$ satisfies the inf-sup condition uniformly with respect to the norm $\|\cdot\|_{\boldsymbol{U}_{h, \theta}}$ if there exists a constant $\beta_{1}>0$ that does not depend on the parameter $\theta$ and the mesh size $h$ such that:

$$
\inf _{\mathcal{V}_{\boldsymbol{h}} \in \boldsymbol{U}_{h}} \sup _{\mathcal{U}_{\boldsymbol{h}} \in \boldsymbol{U}_{h}} \frac{A_{h, \theta}\left(\mathcal{U}_{\boldsymbol{h}}, \mathcal{V}_{\boldsymbol{h}}\right)}{\left\|\mathcal{U}_{\boldsymbol{h}}\right\|_{\boldsymbol{U}_{h, \theta}}\left\|\mathcal{V}_{\boldsymbol{h}}\right\|_{\boldsymbol{U}_{h, \theta}}} \geq \beta_{1}
$$

By the Céa's lemma, see [18], we have

Theorem 3.1 If a discretization (3.22) satisfies

1. consistency, namely (3.23);

2. continuity uniformly, namely (3.24);

3. inf-sup condition uniformly with respect to the norm $\|\cdot\|_{\boldsymbol{U}_{h, \theta}}$, namely (3.25),

then we have

$$
\left\|\mathcal{U}-\mathcal{U}_{\boldsymbol{h}}\right\|_{\boldsymbol{U}_{h, \theta}} \leq C_{1} \inf _{\mathcal{V}_{h} \in \boldsymbol{U}_{h}}\left\|\mathcal{U}-\mathcal{V}_{\boldsymbol{h}}\right\|_{\boldsymbol{U}_{h, \theta}},
$$

where $C_{1}$ is independent of the parameter $\theta$ and the mesh size $h$. Further, we say the discretization (3.22) is uniformly stable. 
Now for the HDG method, the parameter $\theta=\tau$ in (3.22) and the bilinear form is given by (3.11), the space $\boldsymbol{U}_{h}=\boldsymbol{Q}_{h} \times \tilde{V}_{h}, \boldsymbol{U}_{\boldsymbol{h}}=\left(\boldsymbol{p}_{h}, \tilde{u}_{h}\right)$. In the first case, the parameter $\tau=\rho h_{K}$, and the norm $\left\|\mathcal{U}_{\boldsymbol{h}}\right\|_{\boldsymbol{U}_{h, \theta}}^{2}=\left\|\boldsymbol{p}_{h}\right\|_{\mathrm{div}, \rho, h}^{2}+\left\|\tilde{u}_{h}\right\|_{0, \rho, h}^{2}$. In the second case, the parameter $\tau=\rho^{-1} h_{K}^{-1}$ and the norm $\left\|\mathcal{U}_{\boldsymbol{h}}\right\|_{\boldsymbol{U}_{h, \theta}}^{2}=$ $\left\|\boldsymbol{p}_{h}\right\|^{2}+\left\|\tilde{u}_{h}\right\|_{\tilde{1}, \rho, h}^{2}$.

Theorem 3.2 We have two uniform stability results for the HDG method as follows:

1. For any $0<\rho \leq 1$, and for $k \geq 0$, if $\boldsymbol{Q}_{h}=\boldsymbol{Q}_{h}^{k+1}, V_{h}=V_{h}^{k}$ and $\hat{V}_{h}=\hat{V}_{h}^{r}$ where $0 \leq r \leq k+1$, or $\boldsymbol{Q}_{h}=\boldsymbol{Q}_{h}^{k, R T}, V_{h}=V_{h}^{k}$ and $\hat{V}_{h}=\hat{V}_{h}^{r}$ where $0 \leq r \leq k$, then the bilinear form $A_{h}((\cdot, \cdot),(\cdot, \cdot))$ with $\tau=\rho h_{K}$ is uniformly stable with respect to the norms defined by (3.12);

2. Assume that $\nabla_{h} V_{h} \subset \boldsymbol{Q}_{h}$, then there exists a positive constant $\rho_{0}$ such that for any $0<\rho \leq \rho_{0}$ the bilinear form $A_{h}((\cdot, \cdot),(\cdot, \cdot))$ with $\tau=\rho^{-1} h_{K}^{-1}$ is uniformly stable with respect to the norms defined by (3.13).

From part 2 of the above theorem, we have the following corollary:

Corollary 3.3 Assume $\nabla_{h} V_{h} \subset \boldsymbol{Q}_{h}$, then there exists a unique solution $\left(\boldsymbol{p}_{h}, \tilde{u}_{h}\right) \in \boldsymbol{Q}_{h} \times \tilde{V}_{h}$ that satisfies (3.8) with $\tau=\rho^{-1} h_{K}^{-1}$, and there exists a positive constant $\rho_{0}$ such that for any $0<\rho \leq \rho_{0}$ the following estimate holds:

$$
\left\|\boldsymbol{p}_{h}\right\|+\left\|\tilde{u}_{h}\right\|_{\tilde{1}, \rho, h} \leq C_{2}\|f\|_{*, \rho},
$$

where $C_{2}$ is a constant independent of $\rho$ and $h$ and $\|f\|_{*, \rho}=\sup _{\tilde{v}_{h} \in \tilde{V}_{h}} \frac{\left(f, v_{h}\right)_{\tau_{h}}}{\left\|\tilde{v}_{h}\right\|_{\tilde{1}, \rho, h}}$.

Remark 3.4 From the above corollary and the discrete Poincaré-Friedrichs inequalities for piecewise $H^{1}$ functions [19], that is $\left\|v_{h}\right\| \lesssim\left\|\nabla_{h} v_{h}\right\|+\sum_{e \in \mathcal{E}_{h}} h_{e}^{-1}\left\|\llbracket v_{h} \rrbracket\right\|_{0, e}$, we further have $\left\|\boldsymbol{p}_{h}\right\|+\left\|\tilde{u}_{h}\right\|_{\tilde{1}, \rho, h} \leq C_{2}\|f\|$.

Remark 3.5 By the uniform stability results of the HDG method, namely Corollary 3.3, we can prove that the solution of the HDG method converges to the solution of the primal conforming method when the parameter $\rho$ approaches to zero, see Section 5.

Next, for the WG method, the parameter $\theta=\eta$ in (3.22) and the bilinear form is given by (3.19), and the space $\boldsymbol{U}_{h}=\tilde{\boldsymbol{Q}}_{\boldsymbol{h}} \times V_{h}, \boldsymbol{U}_{\boldsymbol{h}}=\left(\tilde{\boldsymbol{p}}_{\boldsymbol{h}}, u_{h}\right)$. In first case, the parameter $\eta=\rho h_{K}$ and the norm $\left\|\mathcal{U}_{\boldsymbol{h}}\right\|_{\boldsymbol{U}_{h, \theta}}^{2}=\left\|\tilde{\boldsymbol{p}}_{h}\right\|_{0, h, \rho}^{2}+$ $\left\|u_{h}\right\|_{1, h, \rho}^{2}$. In the second case, the parameter $\eta=\rho^{-1} h_{K}^{-1}$, and the norm $\left\|\mathcal{U}_{\boldsymbol{h}}\right\|_{\boldsymbol{U}_{h, \theta}}^{2}=\left\|\tilde{\boldsymbol{p}}_{h}\right\|_{\widetilde{\mathrm{div}, \rho, h}}^{2}+\left\|u_{h}\right\|^{2}$.

Theorem 3.6 We have two uniform stability results for the WG method as follows:

1. Assume $\nabla_{h} V_{h} \subset \boldsymbol{Q}_{h}$, then for any $0<\rho \leq 1$ the bilinear form $A_{w}((\cdot, \cdot),(\cdot, \cdot))$ with $\eta=\rho h_{K}$ is uniformly stable with respect to the norms defined by (3.20); 
2. Let $\boldsymbol{R}_{h} \subset H(\operatorname{div}, \Omega) \cap \boldsymbol{Q}_{h}$ be the Raviart-Thomas finite element space. Assume that $\left\{\left[\boldsymbol{R}_{h}\right\} \subset \hat{Q}_{h}\right.$ and $V_{h}=\operatorname{div}_{h} \boldsymbol{Q}_{h}$, then for any $0<\rho \leq 1$ the bilinear form $A_{w}((\cdot, \cdot),(\cdot, \cdot))$ with $\eta=\rho^{-1} h_{K}^{-1}$ is uniformly stable with respect to the norms defined by (3.21).

From part 1 of the above theorem, we have the following corollary:

Corollary 3.7 Assume $\nabla_{h} V_{h} \subset \boldsymbol{Q}_{h}$, then there exists a unique solution $\left(\tilde{\boldsymbol{p}}_{h}, u_{h}\right) \in \tilde{\boldsymbol{Q}}_{h} \times V_{h}$ that satisfies (3.16) with $\eta=\rho h_{K}$, and for any $0<\rho \leq 1$ the following estimates holds:

$$
\left\|\tilde{\boldsymbol{p}}_{h}\right\|_{0, h, \rho}+\left\|u_{h}\right\|_{1, h, \rho} \leq C_{3}\|f\|_{*, \rho}
$$

where $C_{3}$ is a constant uniform with respect to $\rho$ and $h$ and $\|f\|_{*, \rho}=\sup _{v_{h} \in \tilde{V}_{h}} \frac{\left(f, v_{h}\right) \tau_{h}}{\left\|v_{h}\right\|_{1, h, \rho}}$.

Remark 3.8 From the above theorem, we improved the result in [12] by proving the well-posedness of the $W G$ method for any $0<\rho \leq 1$, while in [12] the inf-sup condition for some constant $\rho$ (for example $\rho=1$ ) was proved.

From part 2 of Theorem 3.6, we have the following corollary:

Corollary 3.9 Assume the spaces $\tilde{\boldsymbol{Q}}_{h} \times V_{h}$ satisfy the conditions in part 2 of Theorem 3.6, then there exists a unique solution $\left(\tilde{\boldsymbol{p}}_{h}, u_{h}\right) \in \tilde{\boldsymbol{Q}}_{h} \times V_{h}$ that satisfies (3.16) with $\eta=\rho^{-1} h_{K}^{-1}$, and for any $0<\rho \leq 1$ the following estimates holds:

$$
\left\|\tilde{\boldsymbol{p}}_{h}\right\|_{\widetilde{\mathrm{div}}, h, \rho}+\left\|u_{h}\right\| \leq C_{4}\|f\|
$$

where $C_{4}$ is a uniform constant with respect to $\rho$ and $h$.

Remark 3.10 By the above uniform stability result of the WG method, namely Corollary 3.9, we can prove that the solution of the WG method converges to the solution of the mixed conforming method when the parameter $\rho$ approaches to zero, see Section 5.

\section{Uniform Error Estimates of HDG and WG}

In this section, based on the uniform stability results shown in Section 3, we provide the error analysis for HDG and WG methods and obtain uniformly optimal error estimates for HDG and WG methods.

\subsection{Error Estimate of HDG Method}

Theorem 4.1 Let $(\boldsymbol{p}, u) \in H(\operatorname{div}, \Omega) \times L^{2}(\Omega)$ be the solution of $(1.2)$ and $\boldsymbol{p} \in \boldsymbol{H}^{\boldsymbol{k}+\mathbf{1}}(\boldsymbol{\Omega}), \operatorname{div} \boldsymbol{p} \in H^{k+1}(\Omega), u \in$ $H^{k+1}(\Omega)(k \geq 0)$, and $\left(\boldsymbol{p}_{h}, \tilde{u}_{h}\right) \in \boldsymbol{Q}_{h} \times \tilde{V}_{h}$ be the solution of (3.8) with $\tau=\rho h_{K}$. If we choose the spaces 
$V_{h} \times \boldsymbol{Q}_{h} \times \hat{V}_{h}=V_{h}^{k} \times \boldsymbol{Q}_{h}^{k, R T} \times \hat{V}_{h}^{k}$, then for any $0<\rho \leq 1$ the following estimate holds:

$$
\left\|\boldsymbol{p}-\boldsymbol{p}_{h}\right\|_{\operatorname{div}, \rho, h}+\left\|u-\tilde{u}_{h}\right\|_{0, \rho, h} \leq C_{r, 1} h^{k+1}\left(|\boldsymbol{p}|_{k+1}+|\operatorname{div} \boldsymbol{p}|_{k+1}+|u|_{k+1}\right)
$$

where $C_{r, 1}$ is a constant independent of $h$ and $\rho$.

Proof. From part 1 of Theorem 3.2 and Theorem 3.1, we have

$$
\left\|\boldsymbol{p}-\boldsymbol{p}_{h}\right\|_{\operatorname{div}, \rho, h}+\left\|u-\tilde{u}_{h}\right\|_{0, \rho, h} \lesssim \inf _{\boldsymbol{q}_{h} \in \boldsymbol{Q}_{h}, \tilde{v}_{h} \in \tilde{V}_{h}}\left(\left\|\boldsymbol{p}-\boldsymbol{q}_{h}\right\|_{\operatorname{div}, \rho, h}+\left\|u-\tilde{v}_{h}\right\|_{0, \rho, h}\right) .
$$

Hence we need to estimate:

$$
\inf _{\boldsymbol{q}_{h} \in \boldsymbol{Q}_{h}, \tilde{v}_{h} \in \tilde{V}_{h}}\left(\left\|\boldsymbol{p}-\boldsymbol{q}_{h}\right\|_{\mathrm{div}, \rho, h}+\left\|u-\tilde{v}_{h}\right\|_{0, \rho, h}\right) .
$$

Now we choose $\boldsymbol{q}_{h}=\pi_{h}^{\text {div }} \boldsymbol{p} \in \boldsymbol{Q}_{h}$, where $\pi_{h}^{\text {div }}$ be the interpolation of $\boldsymbol{p}$ into the $H$ (div)-conforming RaviartThomas $(R T)$ finite element space, namely $\boldsymbol{q}_{h} \in \boldsymbol{Q}_{h} \cap H(\operatorname{div}, \Omega)$. Since $\boldsymbol{q}_{h} \cdot \boldsymbol{n}$ is single-valued, then by the approximation property of the $R T$ finite element space, we obtain:

$$
\begin{aligned}
\left\|\boldsymbol{p}-\boldsymbol{q}_{h}\right\|_{\mathrm{div}, \rho, h}^{2}= & \left(c\left(\boldsymbol{p}-\boldsymbol{q}_{h}\right), \boldsymbol{p}-\boldsymbol{q}_{h}\right)_{\mathcal{T}_{h}}+\left(\operatorname{div}\left(\boldsymbol{p}-\boldsymbol{q}_{h}\right), \operatorname{div}\left(\boldsymbol{p}-\boldsymbol{q}_{h}\right)\right)_{\mathcal{T}_{h}} \\
& +\rho^{-1} \sum_{e \in \mathcal{E}_{h}^{i}} h_{e}^{-1}\left\langle\hat{P}_{e}\left(\left[\boldsymbol{p}-\boldsymbol{q}_{h}\right]\right), \hat{P}_{e}\left(\left[\boldsymbol{p}-\boldsymbol{q}_{h}\right]\right)\right\rangle_{e} \\
= & \left(c\left(\boldsymbol{p}-\boldsymbol{q}_{h}\right), \boldsymbol{p}-\boldsymbol{q}_{h}\right)_{\mathcal{T}_{h}}+\left(\operatorname{div}\left(\boldsymbol{p}-\boldsymbol{q}_{h}\right), \operatorname{div}\left(\boldsymbol{p}-\boldsymbol{q}_{h}\right)\right)_{\mathcal{T}_{h}} \\
\lesssim & h^{2 k+2}\left(|\boldsymbol{p}|_{k+1}^{2}+|\operatorname{div} \boldsymbol{p}|_{k+1}^{2}\right) .
\end{aligned}
$$

Further, we choose $v_{h}=Q_{h}(u), \hat{v}_{h}=\left\{Q_{h}(u)\right\}$, where $Q_{h}$ is $L^{2}$ projection from $L^{2}(\Omega)$ to $V_{h}$. Then, by using the approximation of $L^{2}$ projection, trace inequality and noting that $0<\rho \leq 1$, we have:

$$
\begin{aligned}
\left\|u-\tilde{v}_{h}\right\|_{0, \rho, h}^{2} & =\left(u-v_{h}, u-v_{h}\right)_{\mathcal{T}_{h}}+\rho \sum_{e \in \mathcal{E}_{h}^{i}} h_{e}\left\langle u-\hat{v}_{h}, u-\hat{v}_{h}\right\rangle_{e} \\
& =\left(u-Q_{h}(u), u-Q_{h}(u)\right)_{\mathcal{T}_{h}}+\rho \sum_{e \in \mathcal{E}_{h}^{i}} h_{e}\left\langle u-\left\{Q_{h}(u)\right\}, u-\left\{Q_{h}(u)\right\}\right\rangle_{e} \\
& \lesssim\left\|u-Q_{h}(u)\right\|^{2}+\rho \sum_{e \in \mathcal{E}_{h}^{i}} h_{e}\left(h_{e}^{-1}\left\|u-Q_{h}(u)\right\|_{K_{e, 1} \cup K_{e, 2}}^{2}+h_{e}\left\|\nabla_{h}\left(u-Q_{h}(u)\right)\right\|_{K_{e, 1} \cup K_{e, 2}}^{2}\right) \\
& \lesssim\left\|u-Q_{h}(u)\right\|^{2}+h_{e}^{2}\left\|\nabla_{h}\left(u-Q_{h}(u)\right)\right\|^{2} \lesssim h^{2 k+2}|u|_{k+1}^{2},
\end{aligned}
$$

where $K_{e, 1}, K_{e, 2}$ are the elements sharing the edge $e$.

Combining (4.4) and (4.5), we get the desired result.

Theorem 4.2 Let $(\boldsymbol{p}, u) \in \boldsymbol{L}^{\mathbf{2}}(\boldsymbol{\Omega}) \times H^{1}(\Omega)$ be the solution of $(1.2)$ and $\boldsymbol{p} \in \boldsymbol{H}^{\boldsymbol{k + 1}}(\boldsymbol{\Omega}), u \in H^{k+2}(\Omega)$ $(k \geq 0)$, and $\left(\boldsymbol{p}_{h}, \tilde{u}_{h}\right) \in \boldsymbol{Q}_{h} \times \tilde{V}_{h}$ be the solution of (3.8) with $\tau=\rho^{-1} h_{K}^{-1}$. If we choose the spaces 
$V_{h} \times \boldsymbol{Q}_{h} \times \hat{V}_{h}=V_{h}^{k+1} \times \boldsymbol{Q}_{h}^{k} \times \hat{V}_{h}^{k+1}$, then there exists $\rho_{0}>0$ such that for any $0<\rho \leq \rho_{0}$ the following estimate holds:

$$
\left\|\boldsymbol{p}-\boldsymbol{p}_{h}\right\|+\left\|u-\tilde{u}_{h}\right\|_{\tilde{1}, \rho, h} \leq C_{r, 2} h^{k+1}\left(|\boldsymbol{p}|_{k+1}+|u|_{k+2}\right),
$$

where $C_{r, 2}$ is independent of $h$ and $\rho$.

Proof. From part 2 of Theorem 3.2 and Theorem 3.1, we have

$$
\left\|\boldsymbol{p}-\boldsymbol{p}_{h}\right\|+\left\|u-\tilde{u}_{h}\right\|_{\tilde{1}, \rho, h} \lesssim \inf _{\boldsymbol{q}_{h} \in \boldsymbol{Q}_{h}, \tilde{v}_{h} \in \tilde{V}_{h}}\left(\left\|\boldsymbol{p}-\boldsymbol{q}_{h}\right\|+\left\|u-\tilde{v}_{h}\right\|_{\tilde{1}, \rho, h}\right) .
$$

Hence we need to estimate:

$$
\inf _{\boldsymbol{q}_{h} \in \boldsymbol{Q}_{h}, \tilde{v}_{h} \in \tilde{V}_{h}}\left(\left\|\boldsymbol{p}-\boldsymbol{q}_{h}\right\|+\left\|u-\tilde{v}_{h}\right\|_{\tilde{1}, \rho, h}\right) .
$$

Now we choose $\boldsymbol{q}_{h}=Q_{h}(\boldsymbol{p})$, where $Q_{h}$ is $L^{2}$ projection from $\boldsymbol{L}^{2}(\boldsymbol{\Omega})$ to $\boldsymbol{Q}_{h}$. Then, by using the approximation of $L^{2}$ projection, we obtain:

$$
\left\|\boldsymbol{p}-\boldsymbol{q}_{h}\right\| \lesssim h^{k+1}|\boldsymbol{p}|_{k+1} .
$$

Further, we choose $v_{h}=\pi_{h} u$, where $\pi_{h}$ is the interpolation of $u$ to the continuous finite element space, namely $v_{h} \in V_{h} \cap H_{0}^{1}(\Omega)$. Since $v_{h}$ is in $H_{0}^{1}(\Omega)$, we can choose $\hat{v}_{h}$ such that $\left.\hat{v}_{h}\right|_{\partial K}=\left.v_{h}\right|_{\partial K}$, for any $K \in \mathcal{T}_{h}$, then we get the following convergence rate result:

$$
\begin{aligned}
\left\|u-\tilde{v}_{h}\right\|_{\tilde{1}, \rho, h}^{2}= & \left(\nabla_{h}\left(u-v_{h}\right), \nabla_{h}\left(u-v_{h}\right)\right)_{\mathcal{T}_{h}} \\
& +\rho^{-1} \sum_{K \in \mathcal{T}_{h}} h_{K}^{-1}\left\langle\left(u-v_{h}\right)-\left(u-\hat{v}_{h}\right),\left(u-v_{h}\right)-\left(u-\hat{v}_{h}\right)\right\rangle_{\partial K} \\
= & \left(\nabla\left(u-\pi_{h} u\right), \nabla\left(u-\pi_{h} u\right)\right)_{\mathcal{T}_{h}} \lesssim h^{2 k+2}|u|_{k+2}^{2} .
\end{aligned}
$$

Combining (4.9) and (4.10), we get the desired result.

\subsection{Error Estimate of the WG Method}

Theorem 4.3 Let $(\boldsymbol{p}, u) \in \boldsymbol{L}^{\mathbf{2}}(\boldsymbol{\Omega}) \times H^{1}(\Omega)$ be the solution of (1.2) and $\boldsymbol{p} \in \boldsymbol{H}^{\boldsymbol{k}+\mathbf{1}}(\boldsymbol{\Omega}), u \in H^{k+2}(\Omega)$, and $\left(\tilde{\boldsymbol{p}}_{h}, u_{h}\right) \in \tilde{\boldsymbol{Q}}_{h} \times V_{h}$ be the solution of (3.16) with $\eta=\rho h_{K}$. If we choose the spaces $V_{h} \times \boldsymbol{Q}_{h} \times \hat{Q}_{h}=$ $V_{h}^{k+1} \times \boldsymbol{Q}_{h}^{k} \times \hat{Q}_{h}^{k}$, then for any $0<\rho \leq 1$ the following estimate holds:

$$
\left\|\boldsymbol{p}-\tilde{\boldsymbol{p}}_{h}\right\|_{0, h, \rho}+\left\|u-u_{h}\right\|_{1, h, \rho} \leq C_{r, 3} h^{k+1}\left(|\boldsymbol{p}|_{k+1}+|u|_{k+2}\right),
$$

where $C_{r, 3}$ is independent of $h$ and $\rho$.

Proof. From part 1 of Theorem 3.6 and Theorem 3.1, we have

$$
\left\|\boldsymbol{p}-\tilde{\boldsymbol{p}}_{h}\right\|_{0, h, \rho}+\left\|u-u_{h}\right\|_{1, h, \rho} \lesssim \inf _{\tilde{\boldsymbol{q}}_{h} \in \tilde{\boldsymbol{Q}}_{h}, v_{h} \in V_{h}}\left(\left\|\boldsymbol{p}-\tilde{\boldsymbol{q}}_{h}\right\|_{0, h, \rho}+\left\|u-v_{h}\right\|_{1, h, \rho}\right) .
$$


Hence we need to estimate:

$$
\inf _{\tilde{\boldsymbol{q}}_{h} \in \tilde{\boldsymbol{Q}}_{h}, v_{h} \in V_{h}}\left(\left\|\boldsymbol{p}-\tilde{\boldsymbol{q}}_{h}\right\|_{0, h, \rho}+\left\|u-v_{h}\right\|_{1, h, \rho}\right) .
$$

Now for any $K \in \mathcal{T}_{h}$, we choose $\tilde{\boldsymbol{q}}_{h}=\left(\boldsymbol{q}_{h}, \hat{\boldsymbol{q}}_{\boldsymbol{h}}\right)=\left(Q_{h}(\boldsymbol{p}),\left\{Q_{h}(\boldsymbol{p})\right\}\right)$, where $Q_{h}$ is the local $L^{2}$ projection from $L^{2}(\Omega)$ to $\boldsymbol{Q}_{h}$. By the approximation property of the $L^{2}$ projection, trace inequality and noting that $0<\rho \leq 1$, we obtain:

$$
\begin{aligned}
\left\|\boldsymbol{p}-\tilde{\boldsymbol{q}}_{h}\right\|_{0, h, \rho}^{2} & =\left(c\left(\boldsymbol{p}-Q_{h}(\boldsymbol{p})\right), \boldsymbol{p}-Q_{h}(\boldsymbol{p})\right)_{\mathcal{T}_{h}}+\rho \sum_{K \in \mathcal{T}_{h}} h_{K}\left\|\left(\boldsymbol{p}-Q_{h}(\boldsymbol{p})-\left(\boldsymbol{p}-\left\{Q_{h}(\boldsymbol{p})\right\}\right)\right) \cdot \boldsymbol{n}_{K}\right\|_{0, \partial K}^{2} \\
& \lesssim\left\|\boldsymbol{p}-Q_{h}(\boldsymbol{p})\right\|_{\mathcal{T}_{h}}^{2}+\sum_{K \in \mathcal{T}_{h}} h_{K}\left(\left\|\left(\boldsymbol{p}-Q_{h}(\boldsymbol{p})\right) \cdot \boldsymbol{n}_{K}\right\|_{0, \partial K}^{2}+\left\|\left(\boldsymbol{p}-\left\{Q_{h}(\boldsymbol{p})\right\}\right) \cdot \boldsymbol{n}_{K}\right\|_{0, \partial K}^{2}\right) \\
& \lesssim\left\|\boldsymbol{p}-Q_{h}(\boldsymbol{p})\right\|_{\mathcal{T}_{h}}^{2}+\sum_{K \in \mathcal{T}_{h}}\left(\left\|\boldsymbol{p}-Q_{h}(\boldsymbol{p})\right\|_{0, K}^{2}+h_{K}^{2}\left\|\nabla\left(\boldsymbol{p}-Q_{h}(\boldsymbol{p})\right)\right\|_{0, K}^{2}\right) \\
& \lesssim h^{2 k+2}|\boldsymbol{p}|_{k+1}^{2} .
\end{aligned}
$$

Next we choose $v_{h}=\pi_{h} u$, where $\pi_{h}$ is the interpolation of $u$ to the continuous finite element space, namely, $v_{h} \in V_{h} \cap H_{0}^{1}(\Omega)$, we immediately have:

$$
\begin{aligned}
\left\|u-v_{h}\right\|_{1, h, \rho}^{2} & =\left\|\nabla_{h}\left(u-v_{h}\right)\right\|^{2}+\rho^{-1} \sum_{e \in \mathcal{E}_{h}} h_{e}^{-1}\left\|\hat{Q}_{e}\left(\left[u-v_{h}\right]\right)\right\|_{0, e}^{2} \\
& =\left\|\nabla_{h}\left(u-v_{h}\right)\right\|^{2}=\left\|\nabla_{h}\left(u-\pi_{h} u\right)\right\|^{2} \lesssim h^{2 k+2}|u|_{k+2}^{2}
\end{aligned}
$$

Combining (4.14) and (4.15), we get the desired result.

Theorem 4.4 Let $(\boldsymbol{p}, u) \in H(\operatorname{div}, \Omega) \times L^{2}(\Omega)$ be the solution of $(1.2)$ and $\boldsymbol{p} \in \boldsymbol{H}^{\boldsymbol{k}+\mathbf{1}}(\boldsymbol{\Omega}), \operatorname{div} \boldsymbol{p} \in H^{k+1}(\Omega), u \in$ $H^{k+1}(\Omega)$, and $\left(\tilde{\boldsymbol{p}}_{h}, u_{h}\right) \in \tilde{\boldsymbol{Q}}_{h} \times V_{h}$ be the solution of $(3.16)$ with $\eta=\rho^{-1} h_{K}^{-1}$. If we choose the spaces $V_{h} \times \boldsymbol{Q}_{h} \times \hat{Q}_{h}=V_{h}^{k} \times \boldsymbol{Q}_{h}^{k, R T} \times \hat{Q}_{h}^{k}$, then for any $0<\rho \leq 1$ the following estimate holds:

$$
\left\|\boldsymbol{p}-\tilde{\boldsymbol{p}}_{h}\right\|_{\widetilde{\mathrm{div}}, h, \rho}+\left\|u-u_{h}\right\| \leq C_{r, 4} h^{k+1}\left(|\boldsymbol{p}|_{k+1}+|\operatorname{div} \boldsymbol{p}|_{k+1}+|u|_{k+1}\right)
$$

where $C_{r, 4}$ is independent of $h$ and $\rho$.

Proof. From part 2 of Theorem 3.6 and Theorem 3.1, we have

$$
\left\|\boldsymbol{p}-\tilde{\boldsymbol{p}}_{h}\right\|_{\mathrm{div}, h, \rho}+\left\|u-u_{h}\right\| \lesssim \inf _{\tilde{\boldsymbol{q}}_{h} \in \tilde{\boldsymbol{Q}}_{h}, v_{h} \in V_{h}}\left(\left\|\boldsymbol{p}-\tilde{\boldsymbol{q}}_{h}\right\|_{\operatorname{div}, h, \rho}+\left\|u-v_{h}\right\|\right)
$$

We need to estimate:

$$
\inf _{\tilde{\boldsymbol{q}}_{h} \in \tilde{\boldsymbol{Q}}_{h}, v_{h} \in V_{h}}\left(\left\|\boldsymbol{p}-\tilde{\boldsymbol{q}}_{h}\right\|_{\operatorname{div}, h, \rho}+\left\|u-v_{h}\right\|\right) .
$$

Now we choose $\boldsymbol{q}_{h}=\pi_{h}^{\text {div }} \boldsymbol{p} \in \boldsymbol{Q}_{h}$, where $\pi_{h}^{\text {div }}$ is the interpolation of $\boldsymbol{p}$ into the $H($ div)-conforming $R T$ finite element space, namely $\boldsymbol{q}_{h} \in \boldsymbol{Q}_{h} \cap H(\operatorname{div}, \Omega)$. Since $\boldsymbol{q}_{h} \cdot \boldsymbol{n}$ is single-valued, we can choose $\hat{\boldsymbol{q}}_{h}=\left(\boldsymbol{q}_{h} \cdot \boldsymbol{n}\right) \boldsymbol{n}$, 
then by the approximation property of the $R T$ finite element space, we obtain

$$
\begin{aligned}
\left\|\boldsymbol{p}-\tilde{\boldsymbol{q}}_{h}\right\|_{\operatorname{div}, h, \rho}= & \left(c\left(\boldsymbol{p}-\boldsymbol{q}_{h}\right), \boldsymbol{p}-\boldsymbol{q}_{h}\right)_{\mathcal{T}_{h}}+\left(\operatorname{div}\left(\boldsymbol{p}-\boldsymbol{q}_{h}\right), \operatorname{div}\left(\boldsymbol{p}-\boldsymbol{q}_{h}\right)\right)_{\mathcal{T}_{h}} \\
& +\sum_{K \in \mathcal{T}_{h}} \rho^{-1} h_{K}^{-1}\left\|\left(\boldsymbol{p}-\boldsymbol{p}_{h}-\left(\boldsymbol{p}-\hat{\boldsymbol{p}}_{\boldsymbol{h}}\right)\right) \cdot \boldsymbol{n}_{K}\right\|_{0, \partial K}^{2} \\
= & \left(c\left(\boldsymbol{p}-\boldsymbol{q}_{h}\right), \boldsymbol{p}-\boldsymbol{q}_{h}\right)_{\mathcal{T}_{h}}+\left(\operatorname{div}\left(\boldsymbol{p}-\boldsymbol{q}_{h}\right), \operatorname{div}\left(\boldsymbol{p}-\boldsymbol{q}_{h}\right)\right)_{\mathcal{T}_{h}} \\
\lesssim & h^{2 k+2}\left(|\boldsymbol{p}|_{k+1}^{2}+|\operatorname{div} \boldsymbol{p}|_{k+1}^{2}\right) .
\end{aligned}
$$

Next, we choose $v_{h}=Q_{h}(u)$, where $Q_{h}$ is the $L^{2}$ projection from $L^{2}(\Omega)$ to $V_{h}$, and we immediately have:

$$
\left\|u-v_{h}\right\| \lesssim h^{k+1}|u|_{k+1}
$$

Combining (4.19) and (4.20), we get the desired result.

Remark 4.5 We must point out that the error estimates obtained here are uniform with respect to the parameter $\rho$. Namely, all the constants $C_{r, 1}, C_{r, 2}, C_{r, 3}$, and $C_{r, 4}$ are independent of $\rho$. We figure out the following Table 1.

\begin{tabular}{|c|c|c|c|c|}
\hline FEM & norms & $\boldsymbol{U}_{\boldsymbol{h}}$ & parameter $\theta$ & order \\
\hline \multirow{2}{*}{ HDG } & $\left\|\boldsymbol{p}_{h}\right\|_{\mathrm{div}, \rho, h}\left\|\tilde{u}_{h}\right\|_{0, \rho, h}(3.12)$ & $V_{h}^{k} \times \boldsymbol{Q}_{h}^{k, R T} \times \hat{V}_{h}^{k}$ & $\theta=\tau=\rho h_{K}$ & $k+1$ \\
\cline { 2 - 5 } & $\left\|\boldsymbol{p}_{h}\right\|\left\|\tilde{u}_{h}\right\|_{\tilde{1}, \rho, h}(3.13)$ & $V_{h}^{k+1} \times \boldsymbol{Q}_{h}^{k} \times \hat{V}_{h}^{k}$ & $\theta=\tau=\rho^{-1} h_{K}^{-1}$ & $k+1$ \\
\hline \multirow{2}{*}{ WG } & $\left\|\tilde{\boldsymbol{p}}_{h}\right\|_{0, h, \rho}\left\|u_{h}\right\|_{1, h, \rho}(3.20)$ & $V_{h}^{k+1} \times \boldsymbol{Q}_{h}^{k} \times \hat{Q}_{h}^{k}$ & $\theta=\eta=\rho h_{K}$ & $k+1$ \\
\cline { 2 - 5 } & $\left\|\tilde{\boldsymbol{p}}_{h}\right\|_{\widetilde{\mathrm{div}}, h, \rho}\left\|u_{h}\right\|(3.21)$ & $V_{h}^{k} \times \boldsymbol{Q}_{h}^{k, R T} \times \hat{Q}_{h}^{k}$ & $\theta=\eta=\rho^{-1} h_{K}^{-1}$ & $k+1$ \\
\hline
\end{tabular}

Table 1: Convergence of HDG and WG

\section{Relationships between HDG, primal conforming methods and between WG, mixed conforming method}

In this section, as an application of the uniform stability results, we shall discuss the relationships between HDG and primal conforming methods and the relationship between WG and mixed conforming method. The proof for the results of this section are also shown in [20]. For the convenience of reading and the self-consistency of the paper, we show the proof here again. Further, the numerical results verifying the results of this section can be found in [20]. 


\subsection{Primal conforming methods as the limiting case of HDG methods}

For a given mesh, consider the $H^{1}$-conforming subspace $V_{h}^{c}=V_{h} \cap H_{0}^{1}(\Omega) \subset V_{h}$, then the primal conforming methods in the variational form are written as: Find $\left(u_{h}^{c}, \boldsymbol{p}_{h}^{c}\right) \in V_{h}^{c} \times \boldsymbol{Q}_{h}$ such that

$$
\left\{\begin{array}{rlrl}
\left(c \boldsymbol{p}_{h}^{c}, \boldsymbol{q}_{h}\right)_{\mathcal{T}_{h}}+\left(\nabla u_{h}^{c}, \boldsymbol{q}_{h}\right)_{\mathcal{T}_{h}}=\left(\boldsymbol{g}_{1}, \boldsymbol{q}_{h}\right)_{\mathcal{T}_{h}}+\left\langle g_{2}, \boldsymbol{q}_{h} \cdot \boldsymbol{n}\right\rangle_{\partial \mathcal{T}_{h}} & & \forall \boldsymbol{q}_{h} \in \boldsymbol{Q}_{h}, \\
-\left(\boldsymbol{p}_{h}^{c}, \nabla v_{h}^{c}\right)_{\mathcal{T}_{h}}=\left(f, v_{h}^{c}\right)_{\mathcal{T}_{h}} & \forall v_{h}^{c} \in V_{h}^{c},
\end{array}\right.
$$

where $\boldsymbol{g}_{1}=0$ and $g_{2}=0$ when applied to the Poisson equation (1.2).

We try to prove that the HDG methods (3.8) with the stabilization parameter $\tau=\rho^{-1} h_{K}^{-1}$ converge to primal conforming methods (5.1) when $\rho \rightarrow 0$.

First, by $\nabla V_{h}^{c} \subset \nabla_{h} V_{h} \subset Q_{h}$, the well-posedness of the primal conforming methods (cf. [21]) implies that

$$
\left\|\boldsymbol{p}_{h}^{c}\right\|+\left\|u_{h}^{c}\right\|_{1} \leq C_{p}\left(\|f\|_{-1, h}+\sup _{\boldsymbol{q}_{h} \in \boldsymbol{Q}_{h}} \frac{\left(\boldsymbol{g}_{1}, \boldsymbol{q}_{h}\right)_{\mathcal{T}_{h}}+\left\langle g_{2}, \boldsymbol{q}_{h} \cdot \boldsymbol{n}\right\rangle_{\partial \mathcal{T}_{h}}}{\left\|\boldsymbol{q}_{h}\right\|}\right),
$$

where $\|f\|_{-1, h}=\sup _{v_{h}^{c} \neq 0, v_{h}^{c} \in V_{h}^{c}} \frac{\left(f, v_{h}^{c}\right) \mathcal{T}_{h}}{\left\|v_{h}^{c}\right\|_{1}}$.

Recall that the space define on $\mathcal{E}_{h}$ (see (2.5)) of HDG methods is given by

$$
\hat{V}_{h}=\left\{\hat{v}_{h}:\left.\hat{v}_{h}\right|_{e} \in \hat{V}(e), \forall e \in \mathcal{E}_{h}^{i},\left.\hat{v}_{h}\right|_{\mathcal{E}_{h}^{\partial}}=0\right\}
$$

We make the following assumption on the finite element spaces of stabilized hybrid mixed methods.

Assumption 5.1 Assume that the spaces $\boldsymbol{Q}_{h}, V_{h}$ and $\hat{V}_{h}$ satisfy

1. $\nabla_{h} V_{h} \subset \boldsymbol{Q}_{h}$;

2. $\left.\left\{V_{h}\right\}\right|_{e} \subset \hat{V}(e), \forall e \in \mathcal{E}_{h}^{i}$;

3. There exists a constant $C_{p}^{I}$ independent of $h$, such that for any $u_{h} \in V_{h}$,

$$
\inf _{u_{h}^{I} \in V_{h}^{c}}\left(\left\|\left(u_{h}^{I}-u_{h}^{c}\|+\| \nabla_{h}\left(u_{h}^{I}-u_{h}^{c}\right) \|\right) \leq C_{p}^{I} \sum_{e \in \mathcal{E}_{h}} h_{e}^{-1 / 2}\right\| \llbracket \llbracket u_{h} \rrbracket \|_{0, e},\right.
$$

where $V_{h}^{c}=V_{h} \cap H^{1}(\Omega)$.

We note that the first assumption in Assumption 5.1 ensures the well-posedness of the primal conforming methods (5.1). The following example satisfies Assumption 5.1 (see the conforming relatives in [22, 21]).

Example 5.2 $\boldsymbol{Q}_{h}=\boldsymbol{Q}_{h}^{k}, V_{h}=V_{h}^{k+1}, \hat{V}(e)=\mathcal{P}_{k+1}(e)$, for $k \geq 0$. 
For any given $\tau=\rho^{-1} h_{K}^{-1}$, we rewrite the HDG methods (3.8) in the variational form as: Find $\left(\boldsymbol{p}_{h}^{\tau}, u_{h}^{\tau}, \hat{u}_{h}^{\tau}\right) \in \boldsymbol{Q}_{h} \times V_{h} \times \hat{V}_{h}$ such that for any $\left(\boldsymbol{q}_{h}, v_{h}, \hat{v}_{h}\right) \in \boldsymbol{Q}_{h} \times V_{h} \times \hat{V}_{h}$

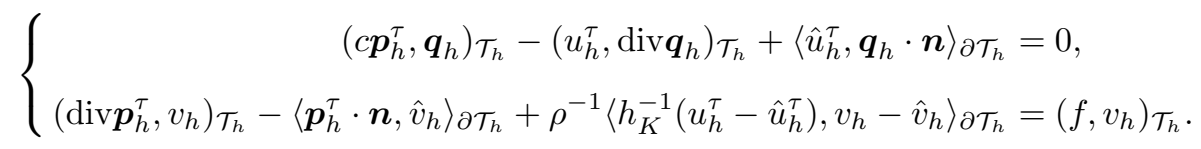

Theorem 5.3 Under the Assumption 5.1, the HDG methods (3.8) with $\tau=\rho^{-1} h_{K}^{-1}$ converge to the primal conforming methods (5.1) as $\rho \rightarrow 0$. More precisely, we have

$$
\left\|\boldsymbol{p}_{h}^{\tau}-\boldsymbol{p}_{h}^{c}\right\|+\left\|u_{h}^{\tau}-u_{h}^{c}\right\|_{1, h} \leq C_{d, 3} \rho^{1 / 2}\|f\|_{-\tilde{1}, \rho, h},
$$

where $C_{d, 3}$ is independent of both mesh size $h$ and $\rho$, and $\|f\|_{-\tilde{1}, \rho, h}=\sup _{\tilde{v}_{h} \in \tilde{V}_{h}} \frac{\left(f, v_{h}\right) \mathcal{T}_{h}}{\left\|\tilde{v}_{h}\right\|_{\tilde{1}, \rho, h}}$.

Proof. From the assumption $\left.\left\{V_{h}\right\}\right|_{e} \subset \hat{V}(e)$, by taking $v_{h}=v_{h}^{c}$ and $\left.\hat{v}_{h}\right|_{e}=\left.v_{h}^{c}\right|_{e}$ in (5.4) and integrating by parts, we see that

$$
-\left(\boldsymbol{p}_{h}^{\tau}, \nabla v_{h}^{c}\right)_{\mathcal{T}_{h}}=\left(f, v_{h}^{c}\right)_{\mathcal{T}_{h}} \quad \forall v_{h}^{c} \in V_{h}^{c}
$$

Subtracting (5.1) from the first equation of (5.4) and (5.6), we have

$$
\left\{\begin{array}{rlrl}
\left(c\left(\boldsymbol{p}_{h}^{\tau}-\boldsymbol{p}_{h}^{c}\right), \boldsymbol{q}_{h}\right)_{\mathcal{T}_{h}}+ & \left(\nabla u_{h}^{\tau}-\nabla u_{h}^{c}, \boldsymbol{q}_{h}\right)_{\mathcal{T}_{h}}=\left\langle u_{h}^{\tau}-\hat{u}_{h}^{\tau}, \boldsymbol{q}_{h} \cdot \boldsymbol{n}\right\rangle_{\partial \mathcal{T}_{h}} & & \forall \boldsymbol{q}_{h} \in \boldsymbol{Q}_{h}, \\
-\left(\boldsymbol{p}_{h}^{\tau}-\boldsymbol{p}_{h}^{c}, \nabla v_{h}^{c}\right)_{\mathcal{T}_{h}}=0 & & \forall v_{h}^{c} \in V_{h}^{c}
\end{array}\right.
$$

Again, for any $u_{h}^{I} \in V_{h}^{c}$, we have

$$
\left\{\begin{aligned}
\left(c\left(\boldsymbol{p}_{h}^{\tau}-\boldsymbol{p}_{h}^{c}\right), \boldsymbol{q}_{h}\right)_{\mathcal{T}_{h}}+ & \left(\nabla u_{h}^{I}-\nabla u_{h}^{c}, \boldsymbol{q}_{h}\right)_{\mathcal{T}_{h}}=\left\langle u_{h}^{\tau}-\hat{u}_{h}^{\tau}, \boldsymbol{q}_{h} \cdot \boldsymbol{n}\right\rangle_{\partial \mathcal{T}_{h}}+\left(\nabla u_{h}^{I}-\nabla u_{h}^{\tau}, \boldsymbol{q}_{h}\right)_{\mathcal{T}_{h}} & & \forall \boldsymbol{q}_{h} \in \boldsymbol{Q}_{h}, \\
& -\left(\boldsymbol{p}_{h}^{\tau}-\boldsymbol{p}_{h}^{c}, \nabla v_{h}^{c}\right)_{\mathcal{T}_{h}}=0 & & \forall v_{h}^{c} \in V_{h}^{c} .
\end{aligned}\right.
$$

Because $\boldsymbol{p}_{h}^{\tau}-\boldsymbol{p}_{h}^{c} \in \boldsymbol{Q}_{h}$ and $v_{h}^{c}-u_{h}^{c} \in V_{h}^{c}$, using (5.2), trace inequality, inverse inequality and Cauchy inequality, we obtain

$$
\begin{aligned}
\left\|\boldsymbol{p}_{h}^{\tau}-\boldsymbol{p}_{h}^{c}\right\|+\left\|u_{h}^{I}-u_{h}^{c}\right\|_{1} & \leq C_{p} \sup _{\boldsymbol{q}_{h} \in \boldsymbol{Q}_{h}} \frac{\left\langle u_{h}^{\tau}-\hat{u}_{h}^{\tau}, \boldsymbol{q}_{h} \cdot \boldsymbol{n}\right\rangle_{\partial \mathcal{T}_{h}}+\left(\nabla u_{h}^{I}-\nabla u_{h}^{\tau}, \boldsymbol{q}_{h}\right)_{\mathcal{T}_{h}}}{\left\|\boldsymbol{q}_{h}\right\|} \\
& \lesssim\left|u_{h}^{I}-u_{h}^{\tau}\right|_{1, h}+\left\langle h^{-1}\left(u_{h}^{\tau}-\hat{u}_{h}^{\tau}\right), u_{h}^{\tau}-\hat{u}_{h}^{\tau}\right\rangle_{\partial \mathcal{T}_{h}}^{1 / 2} .
\end{aligned}
$$

Noting that $\left.\left\{V_{h}\right\}\right|_{e} \subset \hat{V}(e)$, and

$$
\left\langle u_{h}^{\tau}-\hat{u}_{h}^{\tau}, u_{h}^{\tau}-\hat{u}_{h}^{\tau}\right\rangle_{\partial \mathcal{T}_{h}}=2\left\langle\left\{u_{h}^{\tau}-\hat{u}_{h}^{\tau}\right\},\left\{u_{h}^{\tau}-\hat{u}_{h}^{\tau}\right\}\right\rangle_{\mathcal{E}_{h}}+\frac{1}{2}\left\langle\llbracket u_{h}^{\tau} \rrbracket, \llbracket u_{h}^{\tau} \rrbracket\right\rangle_{\mathcal{E}_{h}} .
$$


Therefore, Assumption 5.1, (5.9), and (5.10) imply that

$$
\begin{aligned}
\left\|\boldsymbol{p}_{h}^{\tau}-\boldsymbol{p}_{h}^{c}\right\|+\left\|u_{h}^{\tau}-u_{h}^{c}\right\|_{1, h} & \leq \inf _{u_{h}^{I} \in V_{h}^{c}}\left(\left\|\boldsymbol{p}_{h}^{\tau}-\boldsymbol{p}_{h}^{c}\right\|+\left\|u_{h}^{I}-u_{h}^{c}\right\|_{1}+\left\|u_{h}^{\tau}-u_{h}^{I}\right\|_{1, h}\right) \\
& \lesssim\left\langle h^{-1}\left(u_{h}^{\tau}-\hat{u}_{h}^{\tau}\right), u_{h}^{\tau}-\hat{u}_{h}^{\tau}\right\rangle_{\partial \mathcal{T}_{h}}^{1 / 2}+\inf _{u_{h}^{I} \in V_{h}^{c}}\left\|u_{h}^{\tau}-u_{h}^{I}\right\|_{1, h} \\
& \lesssim\left\langle h^{-1}\left(u_{h}^{\tau}-\hat{u}_{h}^{\tau}\right), u_{h}^{\tau}-\hat{u}_{h}^{\tau}\right\rangle_{\partial \mathcal{T}_{h}}^{1 / 2}+\sum_{e \in \mathcal{E}_{h}} h_{e}^{-1 / 2}\left\|\left[u_{h}\right]\right\|_{0, e} \\
& \lesssim\left\langle h^{-1}\left(u_{h}^{\tau}-\hat{u}_{h}^{\tau}\right), u_{h}^{\tau}-\hat{u}_{h}^{\tau}\right\rangle_{\partial \mathcal{T}_{h}}^{1 / 2} \\
& \lesssim \rho^{1 / 2}\|f\|_{-\tilde{1}, \rho, h},
\end{aligned}
$$

where Corollary 3.3 was used in the last step.

Remark 5.4 From the definition of $\|\cdot\|_{\tilde{1}, \rho, h}$, when $\rho \lesssim 1$, we have

$$
\begin{aligned}
\inf _{\hat{v}_{h} \in \hat{V}_{h}}\left\|\tilde{v}_{h}\right\|_{\tilde{1}, \rho, h}^{2} & =\inf _{\hat{v}_{h} \in \hat{V}_{h}}\left(\nabla v_{h}, \nabla v_{h}\right)_{\mathcal{T}_{h}}+\sum_{K \in \mathcal{T}_{h}} \rho^{-1} h_{K}^{-1}\left\langle v_{h}-\hat{v}_{h}, v_{h}-\hat{v}_{h}\right\rangle_{\partial K} \\
& \simeq\left(\nabla v_{h}, \nabla v_{h}\right)_{\mathcal{T}_{h}}+\rho^{-1} \sum_{e \in \mathcal{E}_{h}} h_{e}^{-1}\left\|\llbracket v_{h} \rrbracket\right\|_{0, e}^{2} \gtrsim\left\|v_{h}\right\|_{1, h} .
\end{aligned}
$$

Hence, when $\rho \lesssim 1$,

$$
\|f\|_{-\tilde{1}, \rho, h}=\sup _{\tilde{v}_{h} \in \tilde{V}_{h}} \frac{\left(f, v_{h}\right)_{\mathcal{T}_{h}}}{\left\|\tilde{v}_{h}\right\|_{\tilde{1}, \rho, h}}=\sup _{v_{h} \in V_{h}} \frac{\left(f, v_{h}\right)_{\mathcal{T}_{h}}}{\inf _{\hat{v}_{h} \in \hat{V}_{h}}\left\|\tilde{v}_{h}\right\|_{\tilde{1}, \rho, h}} \lesssim \sup _{v_{h} \in V_{h}} \frac{\left(f, v_{h}\right)_{\mathcal{T}_{h}}}{\left\|v_{h}\right\|_{1, h}} \lesssim\|f\|,
$$

which means that the solutions of HDG methods converge to those of primal conforming methods with order $\rho^{1 / 2}$ at least.

\subsection{Mixed conforming methods as the limiting case of WG methods}

For a given mesh, consider the $\boldsymbol{H}$ (div)-conforming subspace $\boldsymbol{Q}_{h}^{c}:=\boldsymbol{Q}_{h} \cap \boldsymbol{H}$ (div, $\left.\Omega\right) \subset \boldsymbol{Q}_{h}$, the mixed conforming methods in variational form are written as: Find $\left(\boldsymbol{p}_{h}^{c}, u_{h}^{c}\right) \in \boldsymbol{Q}_{h}^{c} \times V_{h}$ such that

$$
\left\{\begin{aligned}
\left(c \boldsymbol{p}_{h}^{c}, \boldsymbol{q}_{h}^{c}\right)_{\mathcal{T}_{h}}-\left(u_{h}^{c}, \operatorname{div} \boldsymbol{q}_{h}^{c}\right)_{\mathcal{T}_{h}}=\left(g_{1}, \boldsymbol{q}_{h}^{c}\right)_{\mathcal{T}_{h}} & & \forall \boldsymbol{q}_{h}^{c} \in \boldsymbol{Q}_{h}^{c}, \\
\left(\operatorname{div} \boldsymbol{p}_{h}^{c}, v_{h}\right)_{\mathcal{T}_{h}}=\left(f, v_{h}\right)_{\mathcal{T}_{h}}+\left\langle g_{2}, v_{h}\right\rangle_{\partial \mathcal{T}_{h}} & & \forall v_{h} \in V_{h},
\end{aligned}\right.
$$

where $g_{1}=0$ and $g_{2}=0$ when applied to the Poisson equation (1.1).

We will now try to prove that WG methods (3.16) with $\eta=\rho^{-1} h_{K}^{-1}$ converge to mixed conforming methods (5.11) when $\rho \rightarrow 0$.

First, by $V_{h} \subset \operatorname{div} \boldsymbol{Q}_{h}^{c} \subset \operatorname{div}_{h} \boldsymbol{Q}_{h} \subset V_{h}$, the well-posedness of the mixed conforming methods (cf. [23, 24]) implies that

$$
\left\|\boldsymbol{p}_{h}^{c}\right\|_{\boldsymbol{H}(\mathrm{div})}+\left\|v_{h}^{c}\right\| \leq C_{M}\left(\|f\|+\sup _{\boldsymbol{q}_{h}^{c} \in \boldsymbol{Q}_{h}^{c}} \frac{\left(g_{1}, \boldsymbol{q}_{h}^{c}\right)_{\mathcal{T}_{h}}}{\left\|\boldsymbol{q}_{h}^{c}\right\|_{\boldsymbol{H}(\mathrm{div})}}+\sup _{\boldsymbol{v}_{h} \in V_{h}} \frac{\left\langle g_{2}, v_{h}\right\rangle_{\partial \mathcal{T}_{h}}}{\left\|v_{h}\right\|}\right)
$$


Recall that the spaces defined on $\mathcal{E}_{h}$ (see (2.5)) of WG methods are given by

$$
\hat{\boldsymbol{Q}}_{h}=\left\{\hat{\boldsymbol{p}}_{h}:\left.\hat{\boldsymbol{p}}_{h}\right|_{e} \in \hat{Q}(e) \boldsymbol{n}_{e}, \forall e \in \mathcal{E}_{h}\right\}, \quad \hat{Q}_{h}=\left\{\hat{p}_{h}:\left.\hat{p}_{h}\right|_{e}, e \in \hat{Q}(e), \forall e \in \mathcal{E}_{h}\right\}
$$

We make the following assumption on the finite element spaces of WG methods.

Assumption 5.5 Assume that the spaces $\boldsymbol{Q}_{h}, \hat{\boldsymbol{Q}}_{h}$ and $V_{h}$ satisfy

1. $\operatorname{div}_{h} \boldsymbol{Q}_{h}=V_{h}$;

2. $\left.\left\{\left\{\boldsymbol{Q}_{h}\right\}\right\}\right|_{e} \subset \hat{Q}(e), \forall e \in \mathcal{E}_{h}$;

3. There exists a constant $C_{M}^{I}$ independent of $h$, such that for any $\boldsymbol{p}_{h} \in \boldsymbol{Q}_{h}$,

$$
\inf _{\boldsymbol{p}_{h}^{I} \in \boldsymbol{Q}_{h}^{c}}\left(\left\|\boldsymbol{p}_{h}^{I}-\boldsymbol{p}_{h}\right\|+\left\|\operatorname{div}_{h}\left(\boldsymbol{p}_{h}^{I}-\boldsymbol{p}_{h}\right)\right\|\right) \leq C_{M}^{I} \sum_{e \in \mathcal{E}_{h}^{i}} h_{e}^{-1 / 2}\left\|\left[\boldsymbol{p}_{h}\right]\right\|_{0, e},
$$

where $\boldsymbol{Q}_{h}^{c}=\boldsymbol{Q}_{h} \cap \boldsymbol{H}(\operatorname{div} ; \Omega)$.

We note that the first assumption in Assumption 5.5 ensures well-posedness of the mixed conforming methods (5.11). Several examples are given below.

Example 5.6 Raviart-Thomas type: $\boldsymbol{Q}_{h}=\boldsymbol{Q}_{h}^{k, R T}, \hat{Q}(e)=\mathcal{P}_{k}(e), V_{h}=V_{h}^{k}$, for $k \geq 0$.

Example 5.7 Brezzi-Douglas-Marini type: $\boldsymbol{Q}_{h}=\boldsymbol{Q}_{h}^{k+1}, \hat{Q}(e)=\mathcal{P}_{k+1}(e), V_{h}=V_{h}^{k}$, for $k \geq 0$.

Lemma 5.8 If we choose the spaces as in Example 5.7 or Example 5.6, then Assumption 5.5 holds.

Proof. We only sketch the proof of (5.13) in Assumption 5.5. Denote the set of degrees of freedom of RT or BDM element by $D$, see $[23,24]$. We then define $\boldsymbol{p}_{h}^{I}$ as

$$
d\left(\boldsymbol{p}_{h}^{I}\right)=\frac{1}{\left|\mathcal{T}_{d}\right|} \sum_{K \in \mathcal{T}_{d}} d\left(\left.\boldsymbol{p}_{h}\right|_{T}\right) \quad \forall d \in D,
$$

where $\mathcal{T}_{d}$ denotes the set of elements that share the degrees of freedom $d$ and $\left|\mathcal{T}_{d}\right|$ denotes the cardinality of this set. By the standard scaling argument,

$$
\sum_{K \in \mathcal{T}_{h}}\left\|\boldsymbol{p}_{h}^{I}-\boldsymbol{p}_{h}\right\| \lesssim \sum_{e \in \mathcal{E}_{h}^{i}} h_{e}^{1 / 2}\left\|\left[\boldsymbol{p}_{h}\right]\right\|_{0, e}
$$

Then (5.13) follows from the inverse inequality.

For any given $\eta=\rho^{-1} h_{K}^{-1}$, we rewrite the WG methods (3.16) in the variational form as: Find $\left(\boldsymbol{p}_{h}^{\eta}, u_{h}^{\eta}, \hat{\boldsymbol{p}}_{h}^{\eta}\right) \in \boldsymbol{Q}_{h} \times V_{h} \times \hat{\boldsymbol{Q}}_{h}$ such that for any $\left(\boldsymbol{q}_{h}, v_{h}, \hat{\boldsymbol{q}}_{h}\right) \in \boldsymbol{Q}_{h} \times V_{h} \times \hat{\boldsymbol{Q}}_{h}$

$$
\left\{\begin{aligned}
\left(c \boldsymbol{p}_{h}^{\eta}, \boldsymbol{q}_{h}\right)_{\mathcal{T}_{h}}+\rho^{-1}\left\langle h_{K}^{-1}\left(\boldsymbol{p}_{h}^{\eta}-\hat{\boldsymbol{p}}_{h}\right) \cdot \boldsymbol{n},\left(\boldsymbol{q}_{h}-\hat{\boldsymbol{q}}_{h}\right) \cdot \boldsymbol{n}\right\rangle_{\partial \mathcal{T}_{h}}+\left(\nabla u_{h}, q_{h}\right)_{\mathcal{T}_{h}}-\left\langle u_{h}, \hat{\boldsymbol{q}}_{h} \cdot \boldsymbol{n}\right\rangle_{\partial \mathcal{T}_{h}} & =0 \\
-\left(\boldsymbol{p}_{h}^{\eta}, \nabla v_{h}\right)_{\partial \mathcal{T}_{h}}+\left\langle\hat{\boldsymbol{p}}_{h}^{\eta} \cdot \boldsymbol{n}, v_{h}\right\rangle_{\partial \mathcal{T}_{h}} & =\left(f, v_{h}\right) .
\end{aligned}\right.
$$


Theorem 5.9 Under the Assumption 5.5, WG WG methodsmethods (3.16) converge to the mixed conforming methods (5.11) as $\rho \rightarrow 0$ with $\eta=\rho^{-1} h_{K}^{-1}$. More precisely, we have

$$
\left\|\boldsymbol{p}_{h}^{\eta}-\boldsymbol{p}_{h}^{c}\right\|_{\boldsymbol{H}_{h}(\mathrm{div})}+\left\|u_{h}^{\eta}-u_{h}^{c}\right\| \leq C_{w, 3} \rho^{1 / 2}\|f\|,
$$

where $C_{w, 3}$ is independent of both mesh size $h$ and $\rho$.

Proof. From the assumption $\left.\left\{\left\{\boldsymbol{Q}_{h}\right\}\right\}\right|_{e} \subset \hat{Q}(e)$, by taking $\boldsymbol{q}_{h}=\boldsymbol{q}_{h}^{c}$ and $\left.\hat{\boldsymbol{q}}_{h}\right|_{e}=\left(\boldsymbol{q}_{h}^{c} \cdot \boldsymbol{n}_{e}\right) \boldsymbol{n}_{e}$ in (5.14) and integrating by parts, we see that $\left(\boldsymbol{p}_{h}^{\eta}, u_{h}^{\eta}\right)$ satisfies

$$
\left(c \boldsymbol{p}_{h}^{\eta}, \boldsymbol{q}_{h}^{c}\right)_{\mathcal{T}_{h}}-\left(u_{h}^{\eta}, \operatorname{div} \boldsymbol{q}_{h}^{c}\right)_{\mathcal{T}_{h}}=0 \quad \forall \boldsymbol{q}_{h}^{c} \in \boldsymbol{Q}_{h}^{c} .
$$

Subtracting (5.11) from (5.16) and the second equation of (5.14), we have

$$
\left\{\begin{array}{rlrl}
\left(c\left(\boldsymbol{p}_{h}^{\eta}-\boldsymbol{p}_{h}^{c}\right), \boldsymbol{q}_{h}^{c}\right)_{\mathcal{T}_{h}}-\left(u_{h}^{\eta}-u_{h}^{c}, \operatorname{div} \boldsymbol{q}_{h}^{c}\right)_{\mathcal{T}_{h}} & =0 & & \forall \boldsymbol{q}_{h}^{c} \in \boldsymbol{Q}_{h}^{c}, \\
\left(\operatorname{div}\left(\boldsymbol{p}_{h}^{\eta}-\boldsymbol{p}_{h}^{c}\right), v_{h}\right)_{\mathcal{T}_{h}}=\left\langle\left(\boldsymbol{p}_{h}^{\eta}-\hat{\boldsymbol{p}}_{h}^{\eta}\right) \cdot \boldsymbol{n}, v_{h}\right\rangle_{\partial \mathcal{T}_{h}} & & \forall v_{h} \in V_{h} .
\end{array}\right.
$$

Noting that $\boldsymbol{p}_{h}^{\eta} \notin \boldsymbol{Q}_{h}^{c}$, we have that, for any $\boldsymbol{p}_{h}^{I} \in \boldsymbol{Q}_{h}^{c}$,

$$
\begin{cases}\left(c\left(\boldsymbol{p}_{h}^{I}-\boldsymbol{p}_{h}^{c}\right), \boldsymbol{q}_{h}^{c}\right)_{\mathcal{T}_{h}}-\left(u_{h}^{\eta}-u_{h}^{c}, \operatorname{div} \boldsymbol{q}_{h}^{c}\right)_{\mathcal{T}_{h}}=\left(c\left(\boldsymbol{p}_{h}^{I}-\boldsymbol{p}_{h}^{\eta}\right), \boldsymbol{q}_{h}^{c}\right)_{\mathcal{T}_{h}} & \forall \boldsymbol{q}_{h}^{c} \in \boldsymbol{Q}_{h}^{c}, \\ \left(\operatorname{div}\left(\boldsymbol{p}_{h}^{I}-\boldsymbol{p}_{h}^{c}\right), v_{h}\right)_{\mathcal{T}_{h}}=\left\langle\left(\boldsymbol{p}_{h}^{\eta}-\hat{\boldsymbol{p}}_{h}^{\eta}\right) \cdot \boldsymbol{n}, v_{h}\right\rangle_{\partial \mathcal{T}_{h}}+\left(\operatorname{div}\left(\boldsymbol{p}_{h}^{I}-\boldsymbol{p}_{h}^{\eta}\right), v_{h}\right)_{\mathcal{T}_{h}} & \forall v_{h} \in V_{h} .\end{cases}
$$

Because $\left(\boldsymbol{p}_{h}^{I}-\boldsymbol{p}_{h}^{c}\right) \in \boldsymbol{Q}_{h}^{c},\left(u_{h}^{\eta}-u_{h}^{c}\right) \in V_{h}$, by the well-posedness of the mixed conforming methods (5.12), trace inequality, inverse inequality and Cauchy inequality, we have

$$
\begin{aligned}
& \left\|\boldsymbol{p}_{h}^{I}-\boldsymbol{p}_{h}^{c}\right\|_{\boldsymbol{H}(\mathrm{div})}+\left\|u_{h}^{\eta}-u_{h}^{c}\right\| \\
\leq & C_{M}\left(\sup _{\boldsymbol{q}_{h}^{c} \in \boldsymbol{Q}_{h}^{c}} \frac{\left(c\left(\boldsymbol{p}_{h}^{I}-\boldsymbol{p}_{h}^{\eta}\right), \boldsymbol{q}_{h}^{c}\right)_{\mathcal{T}_{h}}}{\left\|\boldsymbol{q}_{h}^{c}\right\|_{\boldsymbol{H}(\operatorname{div})}}+\sup _{v_{h} \in V_{h}} \frac{\left\langle\left(\boldsymbol{p}_{h}^{\eta}-\hat{\boldsymbol{p}}_{h}^{\eta}\right) \cdot \boldsymbol{n}, v_{h}\right\rangle_{\partial \mathcal{T}_{h}}+\left(\operatorname{div}\left(\boldsymbol{p}_{h}^{I}-\boldsymbol{p}_{h}^{\eta}\right), v_{h}\right)_{\mathcal{T}_{h}}}{\left\|v_{h}\right\|}\right) \\
\lesssim & \left\|\boldsymbol{p}_{h}^{I}-\boldsymbol{p}_{h}^{\eta}\right\|+\left\|\operatorname{div}_{h}\left(\boldsymbol{p}_{h}^{I}-\boldsymbol{p}_{h}^{\eta}\right)\right\|+\left\langle h^{-1}\left(\boldsymbol{p}_{h}^{\eta}-\hat{\boldsymbol{p}}_{h}^{\eta}\right) \cdot \boldsymbol{n},\left(\boldsymbol{p}_{h}^{\eta}-\hat{\boldsymbol{p}}_{h}^{\eta}\right) \cdot \boldsymbol{n}\right\rangle_{\partial \mathcal{T}_{h}}^{1 / 2} .
\end{aligned}
$$

Hence, by Assumption 5.5 and inverse inequality, we have

$$
\begin{aligned}
\left\|\boldsymbol{p}_{h}^{\eta}-\boldsymbol{p}_{h}^{c}\right\|_{\boldsymbol{H}(\mathrm{div})}+\left\|u_{h}^{\eta}-u_{h}^{c}\right\| & \lesssim\left\langle h^{-1}\left(\boldsymbol{p}_{h}^{\eta}-\hat{\boldsymbol{p}}_{h}^{\eta}\right) \cdot \boldsymbol{n},\left(\boldsymbol{p}_{h}^{\eta}-\hat{\boldsymbol{p}}_{h}^{\eta}\right) \cdot \boldsymbol{n}\right\rangle_{\partial \mathcal{T}_{h}}^{1 / 2}+\inf _{\boldsymbol{p}_{h}^{I} \in \boldsymbol{Q}_{h}^{c}}\left(\left\|\boldsymbol{p}_{h}^{I}-\boldsymbol{p}_{h}^{\eta}\right\|+\left\|\operatorname{div}_{h}\left(\boldsymbol{p}_{h}^{I}-\boldsymbol{p}_{h}^{\eta}\right)\right\|\right) \\
& \lesssim\left\langle h^{-1}\left(\boldsymbol{p}_{h}^{\eta}-\hat{\boldsymbol{p}}_{h}^{\eta}\right) \cdot \boldsymbol{n},\left(\boldsymbol{p}_{h}^{\eta}-\hat{\boldsymbol{p}}_{h}^{\eta}\right) \cdot \boldsymbol{n}\right\rangle_{\partial \mathcal{T}_{h}}^{1 / 2}+\sum_{e \in \mathcal{E}_{h}^{i}} h_{e}^{-1 / 2}\left\|\left[\boldsymbol{p}_{h}^{\eta}\right]\right\|_{0, e} .
\end{aligned}
$$

From the fact that

$$
\left\langle\left(\boldsymbol{p}_{h}^{\eta}-\hat{\boldsymbol{p}}_{h}^{\eta}\right) \cdot \boldsymbol{n},\left(\boldsymbol{p}_{h}^{\eta}-\hat{\boldsymbol{p}}_{h}^{\eta}\right) \cdot \boldsymbol{n}\right\rangle_{\partial \mathcal{T}_{h}}=2\left\langle\left\{\left\{\boldsymbol{p}_{h}^{\eta}-\hat{\boldsymbol{p}}_{h}^{\eta}\right\},\left\{\left\{\boldsymbol{p}_{h}^{\eta}-\hat{\boldsymbol{p}}_{h}^{\eta}\right\}\right\rangle_{\mathcal{E}_{h}}+\frac{1}{2}\left\langle\left[\boldsymbol{p}_{h}^{\eta}\right],\left[\boldsymbol{p}_{h}^{\eta}\right]\right\rangle_{\mathcal{E}_{h}},\right.\right.
$$

we obtain

$$
\begin{aligned}
\left\|\boldsymbol{p}_{h}^{\eta}-\boldsymbol{p}_{h}^{c}\right\|_{\boldsymbol{H}(\mathrm{div})}+\left\|u_{h}^{\eta}-u_{h}^{c}\right\| & \lesssim\left\langle h^{-1}\left(\boldsymbol{p}_{h}^{\eta}-\hat{\boldsymbol{p}}_{h}^{\eta}\right) \cdot \boldsymbol{n},\left(\boldsymbol{p}_{h}^{\eta}-\hat{\boldsymbol{p}}_{h}^{\eta}\right) \cdot \boldsymbol{n}\right\rangle_{\partial \mathcal{T}_{h}}^{1 / 2}+\sum_{e \in \mathcal{E}_{h}^{i}} h_{e}^{-1 / 2}\left\|\left[\boldsymbol{p}_{h}^{\eta}\right]\right\|_{0, e} \\
& \lesssim\left\langle h^{-1}\left(\boldsymbol{p}_{h}^{\eta}-\hat{\boldsymbol{p}}_{h}^{\eta}\right) \cdot \boldsymbol{n},\left(\boldsymbol{p}_{h}^{\eta}-\hat{\boldsymbol{p}}_{h}^{\eta}\right) \cdot \boldsymbol{n}\right\rangle_{\partial \mathcal{T}_{h}}^{1 / 2} \lesssim \rho^{1 / 2}\|f\|,
\end{aligned}
$$

where we used Corollary 3.9 in the last step. This completes the proof. 


\section{Analysis of HDG and WG}

In this section, we present the analysis of HDG and WG methods and hence prove the uniformly well-posed results of the HDG and WG methods provided in Section 3. Namely, we prove the Theorem 3.2 for HDG methods and the Theorem 3.6 for WG methods. That means we need to prove that the HDG and WG methods satisfy the consistency, the uniform continuity and the inf-sup condition uniformly with respect to the corresponding norms.

Lemma 6.1 Both the HDG methods and WG methods are consistent.

Proof. By the verification of (3.23), the proof is obvious.

\subsection{Proof for Part 1 of Theorem 3.2}

Theorem 6.2 For any $0<\rho \leq 1$, the bilinear form $A_{h}\left(\left(\boldsymbol{p}_{h}, \tilde{u}_{h}\right),\left(\boldsymbol{q}_{h}, \tilde{v}_{h}\right)\right)$ is uniformly continuous.

Proof. The boundedness of $a_{h}\left(\boldsymbol{p}_{h}, \boldsymbol{q}_{h}\right)$ is obvious. Before we discuss the boundedness of $b_{h}\left(\boldsymbol{q}_{h}, \tilde{u}_{h}\right)$, by $(2.2)$ and noting $\llbracket \hat{u}_{h} \rrbracket=0$, we rewrite $b_{h}\left(\boldsymbol{q}_{h}, \tilde{u}_{h}\right)$ as:

$$
b_{h}\left(\boldsymbol{q}_{h}, \tilde{u}_{h}\right)=-\left(u_{h}, \operatorname{div}_{h} \boldsymbol{q}_{h}\right)_{\mathcal{T}_{h}}+\sum_{K \in \mathcal{T}_{h}}\left\langle\hat{u}_{h}, \boldsymbol{q}_{h} \cdot \boldsymbol{n}_{K}\right\rangle_{\partial K}=-\left(u_{h}, \operatorname{div}_{h} \boldsymbol{q}_{h}\right)_{\mathcal{T}_{h}}+\sum_{e \in \mathcal{E}_{h}^{i}}\left\langle\hat{u}_{h},\left[\boldsymbol{q}_{h}\right]\right\rangle_{e} .
$$

Now we show the boundedness of $b_{h}\left(\boldsymbol{q}_{h}, \tilde{u}_{h}\right)$ here. By (6.1) and the definition of $\hat{P}_{e}$, we have:

$$
\begin{aligned}
b_{h}\left(\boldsymbol{q}_{h}, \tilde{u}_{h}\right) & =-\left(u_{h}, \operatorname{div}_{h} \boldsymbol{q}_{h}\right)_{\mathcal{T}_{h}}+\left\langle\hat{u}_{h},\left[\boldsymbol{q}_{h}\right]\right\rangle_{\mathcal{E}_{h}^{i}} \\
& =-\left(u_{h}, \operatorname{div}_{h} \boldsymbol{q}_{h}\right)_{\mathcal{T}_{h}}+\left\langle\hat{u}_{h}, \hat{P}_{e}\left(\left[\boldsymbol{q}_{h}\right]\right)\right\rangle_{\mathcal{E}_{h}^{i}} \\
& \leq\left\|\operatorname{div}_{h} \boldsymbol{q}_{h}\right\|\left\|u_{h}\right\|+\left(\rho^{-1} \sum_{e \in \mathcal{E}_{h}^{i}} h_{e}^{-1}\left\langle\hat{P}_{e}\left(\left[\boldsymbol{q}_{h}\right]\right), \hat{P}_{e}\left(\left[\boldsymbol{q}_{h}\right]\right)\right\rangle_{e}\right)^{\frac{1}{2}}\left(\rho \sum_{e \in \mathcal{E}_{h}^{i}} h_{e}\left\langle\hat{u}_{h}, \hat{u}_{h}\right\rangle_{e}\right)^{\frac{1}{2}} \\
& \leq\left\|\boldsymbol{q}_{h}\right\|_{\operatorname{div}, \rho, h}\left\|\tilde{u}_{h}\right\|_{0, \rho, h},
\end{aligned}
$$

which proves the boundedness of $b_{h}\left(\boldsymbol{q}_{h}, \tilde{u}_{h}\right)$.

Next we prove the boundedness of $c_{h}\left(\tilde{u}_{h}, \tilde{v}_{h}\right)$.

By the Cauchy inequality, we have:

$$
\begin{aligned}
\left|c_{h}\left(\tilde{u}_{h}, \tilde{v}_{h}\right)\right| & =\rho \sum_{K \in \mathcal{T}_{h}} h_{K}\left\langle u_{h}-\hat{u}_{h}, v_{h}-\hat{v}_{h}\right\rangle_{\partial K} \\
& \leq\left(\rho \sum_{K \in \mathcal{T}_{h}} h_{K}\left\langle u_{h}-\hat{u}_{h}, u_{h}-\hat{u}_{h}\right\rangle_{\partial K}\right)^{\frac{1}{2}}\left(\rho \sum_{K \in \mathcal{T}_{h}} h_{K}\left\langle v_{h}-\hat{v}_{h}, v_{h}-\hat{v}_{h}\right\rangle_{\partial K}\right)^{\frac{1}{2}}
\end{aligned}
$$


By the trace inequality, inverse inequality, and noting that $0<\rho \leq 1$, we have:

$$
\begin{aligned}
& \rho \sum_{K \in \mathcal{T}_{h}} h_{K}\left\langle u_{h}-\hat{u}_{h}, u_{h}-\hat{u}_{h}\right\rangle_{\partial K} \\
= & \rho \sum_{K \in \mathcal{T}_{h}} h_{K}\left(\left\langle u_{h}, u_{h}\right\rangle_{\partial K}-2\left\langle u_{h}, \hat{u}_{h}\right\rangle_{\partial K}+\left\langle\hat{u}_{h}, \hat{u}_{h}\right\rangle_{\partial K}\right) \\
\leq & 2 \rho \sum_{K \in \mathcal{T}_{h}} h_{K}\left(\left\langle u_{h}, u_{h}\right\rangle_{\partial K}+\left\langle\hat{u}_{h}, \hat{u}_{h}\right\rangle_{\partial K}\right) \\
\lesssim & 2 \rho \sum_{K \in \mathcal{T}_{h}} h_{K}\left\langle u_{h}, u_{h}\right\rangle_{\partial K}+4 \rho \sum_{K \in \mathcal{E}_{h}^{i}} h_{e}\left\langle\hat{u}_{h}, \hat{u}_{h}\right\rangle_{e} \\
\lesssim & 2 \sum_{K \in \mathcal{T}_{h}}\left(u_{h}, u_{h}\right)_{K}+4 \rho \sum_{K \in \mathcal{E}_{h}^{i}} h_{e}\left\langle\hat{u}_{h}, \hat{u}_{h}\right\rangle_{e} \leq 4\left\|\tilde{u}_{h}\right\|_{0, \rho, h}^{2} .
\end{aligned}
$$

Similarly, we have:

$$
\rho \sum_{K \in \mathcal{T}_{h}} h_{K}\left\langle v_{h}-\hat{v}_{h}, v_{h}-\hat{v}_{h}\right\rangle_{\partial K} \lesssim\left\|\tilde{v}_{h}\right\|_{0, \rho, h}^{2}
$$

Hence, we obtain $\left|c_{h}\left(\tilde{u}_{h}, \tilde{v}_{h}\right)\right| \lesssim\left\|\tilde{u}_{h}\right\|_{0, \rho, h}\left\|\tilde{v}_{h}\right\|_{0, \rho, h}$.

We denote

$$
\operatorname{Ker}(B)=\left\{\boldsymbol{q}_{h} \in \boldsymbol{Q}_{h}: b_{h}\left(\boldsymbol{q}_{h}, \tilde{u}_{h}\right)=0, \forall \tilde{u}_{h} \in \tilde{V}_{h}\right\}
$$

Then, we have the coercivity of $a_{h}(\cdot, \cdot)$ on the $\operatorname{Ker}(B)$ as follows:

Theorem 6.3 Assume that $\operatorname{div}_{h} \boldsymbol{Q}_{h} \subset V_{h}$, then

$$
a_{h}\left(\boldsymbol{p}_{h}, \boldsymbol{p}_{h}\right) \geq\left\|\boldsymbol{p}_{h}\right\|_{\mathrm{div}, \rho, h}^{2}, \quad \forall \boldsymbol{p}_{h} \in \operatorname{Ker}(B) .
$$

Proof. Since

$$
\operatorname{Ker}(B)=\left\{\boldsymbol{q}_{h} \in \boldsymbol{Q}_{h}: b_{h}\left(\boldsymbol{q}_{h}, \tilde{u}_{h}\right)=0, \forall \tilde{u}_{h} \in \tilde{V}_{h}\right\},
$$

then by (6.1) and under the assumption that $\operatorname{div}_{h} \boldsymbol{Q}_{h} \subset V_{h}$, we have:

$$
\begin{aligned}
\operatorname{Ker}(B) & =\left\{\boldsymbol{q}_{h} \in \boldsymbol{Q}_{h}:-\left(u_{h}, \operatorname{div} \boldsymbol{q}_{h}\right)_{\mathcal{T}_{h}}+\left\langle\hat{u}_{h},\left[\boldsymbol{q}_{h}\right]\right\rangle_{\mathcal{E}_{h}^{i}}=0, \forall \tilde{u}_{h} \in \tilde{V}_{h}\right\} \\
& =\left\{\boldsymbol{q}_{h} \in \boldsymbol{Q}_{h}:-\left(u_{h}, \operatorname{div} \boldsymbol{q}_{h}\right)_{\mathcal{T}_{h}}+\left\langle\hat{u}_{h}, \hat{P}_{e}\left(\left[\boldsymbol{q}_{h}\right]\right)\right\rangle_{\mathcal{E}_{h}^{i}}=0, \forall \tilde{u}_{h} \in \tilde{V}_{h}\right\} \\
& =\left\{\boldsymbol{q}_{h} \in \boldsymbol{Q}_{h}: \operatorname{div}_{h} \boldsymbol{q}_{h}=0, \hat{P}_{e}\left(\left[\boldsymbol{q}_{h}\right]\right)=0\right\} .
\end{aligned}
$$

Hence, by the definition of $\left\|\boldsymbol{q}_{h}\right\|_{\text {div }, \rho, h}$, we obtain $a_{h}\left(\boldsymbol{p}_{h}, \boldsymbol{p}_{h}\right) \geq\left\|\boldsymbol{p}_{h}\right\|_{\text {div }, \rho, h}^{2}, \forall \boldsymbol{p}_{h} \in \operatorname{Ker}(B)$.

Lemma 6.4 Given the edges (faces) $e_{1}, e_{2}, \cdots, e_{d+1}$ of the simplex $K$ and functions $\boldsymbol{q} \in \boldsymbol{L}^{2}(\boldsymbol{K})$ and $\hat{\zeta}_{i} \in$ $L^{2}\left(e_{i}\right), i=1, \cdots, d+1$, there is a unique function $\boldsymbol{z} \in \mathcal{P}_{\boldsymbol{r}}(\boldsymbol{K}) \oplus \boldsymbol{x} \mathcal{P}_{r}(K), r \geq 0$ such that,

$$
\begin{aligned}
& (\boldsymbol{z}-\boldsymbol{q}, \boldsymbol{p})_{K}=0, \quad \forall \boldsymbol{p} \in \mathcal{P}_{\boldsymbol{r}-\mathbf{1}}(\boldsymbol{K}), \\
& \left(\boldsymbol{z} \cdot \boldsymbol{n}_{i}-\hat{\zeta}_{i}, \hat{v}\right)_{e_{i}}=0, \quad \forall \hat{v} \in \mathcal{P}_{r}\left(e_{i}\right), i=1, \cdots, d+1,
\end{aligned}
$$


where $\boldsymbol{n}_{i}$ is the outward normal unit vector of $e_{i}$. Moreover:

$$
\|\boldsymbol{z}\|_{0, K} \leq C_{d, r}\left(\|\boldsymbol{q}\|_{0, K}+h_{K}^{1 / 2} \sum_{i=1}^{d+1}\left\|\hat{\zeta}_{i}\right\|_{0, e_{i}}\right),
$$

where $C_{d, r}$ depends only on $d, r$, and the shape regular constant.

Proof. Similar to the definition of the local Raviart-Thomas finite element, the well-posedness of $\boldsymbol{z}$ is obvious. Then, from a simple scaling argument, the estimate is desired.

Let $\tilde{\mathcal{P}}_{\boldsymbol{r}-\mathbf{1}}(\boldsymbol{K})=\left(\tilde{\mathcal{P}}_{r-1}(K)\right)^{d}$ be the vector homogeneous polynomials of degree $r-1$. Similar to Lemma 6.4, we also have:

Lemma 6.5 Given the edges (faces) $e_{1}, e_{2}, \cdots, e_{d+1}$ of the simplex $K$ and functions $\boldsymbol{q} \in \boldsymbol{L}^{\mathbf{2}}(\boldsymbol{K})$ and $\hat{\zeta}_{i} \in$ $L^{2}\left(e_{i}\right), i=1, \cdots, d+1$, there is a unique function $\boldsymbol{z} \in \mathcal{P}_{\boldsymbol{r}}(\boldsymbol{K}), r \geq 1$ such that,

$$
\begin{aligned}
& (\boldsymbol{z}-\boldsymbol{q}, \boldsymbol{p})_{K}=0, \quad \forall \boldsymbol{p} \in \mathcal{P}_{\boldsymbol{r - 2}}(\boldsymbol{K}) \oplus \mathcal{S}_{\boldsymbol{r - 1}}(\boldsymbol{K}), \\
& \left\langle\boldsymbol{z} \cdot \boldsymbol{n}_{i}-\hat{\zeta}_{i}, \hat{v}\right\rangle_{e_{i}}=0, \quad \forall \hat{v} \in \mathcal{P}_{r}\left(e_{i}\right), i=1, \cdots, d+1,
\end{aligned}
$$

where $\boldsymbol{n}_{i}$ is the outward normal unit vector of $e_{i}$ and $\mathcal{S}_{\boldsymbol{r - 1}}(\boldsymbol{K})=\left\{\boldsymbol{v} \in \tilde{\mathcal{P}}_{\boldsymbol{r - 1}}(\boldsymbol{K}): \boldsymbol{x} \cdot \boldsymbol{v}=0\right\}$. Moreover:

$$
\|\boldsymbol{z}\|_{0, K} \leq C_{d, r}\left(\|\boldsymbol{q}\|_{0, K}+h_{K}^{1 / 2} \sum_{i=1}^{d+1}\left\|\hat{\zeta}_{i}\right\|_{0, e_{i}}\right),
$$

where $C_{d, r}$ depends only on $d, r$, and the shape regular constant.

Proof. Similar to the definition of the local Brezzi-Douglas-Marini $(B D M)$ finite element, the wellposedness of $\boldsymbol{z}$ is obvious. From a simple scaling argument, the estimate is desired.

Now we consider the inf-sup condition of $b_{h}\left(\boldsymbol{q}_{h}, \tilde{u}_{h}\right)$.

Theorem 6.6 For $k \geq 1$, assume that $\boldsymbol{Q}_{h}=\boldsymbol{Q}_{h}^{k}, V_{h}=V_{h}^{k-1}$ and $\hat{V}_{h}=\hat{V}_{h}^{r}$, where $0 \leq r \leq k$, or $\boldsymbol{Q}_{h}=$ $\boldsymbol{Q}_{h}^{k, R T}, V_{h}=V_{h}^{k-1}$ and $\hat{V}_{h}=\hat{V}_{h}^{r}$, where $0 \leq r \leq k-1$, then we have:

$$
\inf _{\tilde{u}_{h} \in \tilde{V}_{h}} \sup _{\boldsymbol{q}_{h} \in \boldsymbol{Q}_{h}} \frac{b_{h}\left(\boldsymbol{q}_{h}, \tilde{u}_{h}\right)}{\left\|\boldsymbol{q}_{h}\right\|_{\operatorname{div}, \rho, h}\left\|\tilde{u}_{h}\right\|_{0, \rho, h}} \geq \beta_{2},
$$

where $\beta_{2}>0$ is a constant independent of $\rho$ and mesh size $h$.

Proof. Here we only give the proof under the assumption $\boldsymbol{Q}_{h}=\boldsymbol{Q}_{h}^{k}, V_{h}=V_{h}^{k-1}$ and $\hat{V}_{h}=\hat{V}_{h}^{r}$. The other case is similar.

For any $\tilde{u}_{h} \in \tilde{V}_{h}$, namely for any $u_{h} \in V_{h}, \hat{u}_{h} \in \hat{V}_{h}$, we need to construct a $\boldsymbol{q}_{h} \in \boldsymbol{Q}_{h}$, such that:

$$
b_{h}\left(\boldsymbol{q}_{h}, \tilde{u}_{h}\right)=\left\|\tilde{u}_{h}\right\|_{0, \rho, h}^{2} \text { and }\left\|\boldsymbol{q}_{h}\right\|_{\mathrm{div}, \rho, h} \lesssim\left\|\tilde{u}_{h}\right\|_{0, \rho, h} .
$$


We define $\boldsymbol{z}_{h}$ piecewisely on any $K$, namely $\boldsymbol{z}_{h} \in Q_{h},\left.\boldsymbol{z}_{h}\right|_{K}=\boldsymbol{z}_{K}$ and $\boldsymbol{z}_{K} \in \boldsymbol{P}_{\boldsymbol{r}}(\boldsymbol{K})$ is defined as follows

$$
\begin{aligned}
& \left(\boldsymbol{z}_{K}, \boldsymbol{p}\right)_{K}=0, \quad \forall \boldsymbol{p} \in \mathcal{P}_{\boldsymbol{r - 2}}(\boldsymbol{K}) \oplus \mathcal{S}_{\boldsymbol{r - 1}}(\boldsymbol{K}) \\
& \left\langle\boldsymbol{z}_{K} \cdot \boldsymbol{n}_{i}-\frac{\rho h_{e_{i}} \hat{u}_{h}}{2}, \hat{v}\right\rangle_{e_{i}}=0, \quad \forall \hat{v} \in \mathcal{P}_{r}\left(e_{i}\right), i=1, \cdots, d+1
\end{aligned}
$$

Then, by Lemma 6.5, we have:

$$
\left\|\boldsymbol{z}_{h}\right\|^{2} \lesssim \rho^{2} h_{K} h_{e}^{2} \sum_{e \in \mathcal{E}_{h}^{i}}\left\|\hat{u}_{h}\right\|_{0, e}^{2}
$$

In fact, we also have that for any $e \in \mathcal{E}_{h}^{i}$,

$$
\left.\left[\boldsymbol{z}_{h}\right]\right|_{e}=\left.\rho h_{e} \hat{u}_{h}\right|_{e}
$$

Next, noting that $\operatorname{div} \boldsymbol{z}_{K} \in \mathcal{P}_{r-1}(K)$, then for $-d i v_{h} \boldsymbol{z}_{h}-u_{h}$, there exists $\boldsymbol{r}_{h} \in H(\operatorname{div}, \Omega) \cap \boldsymbol{Q}_{h}$ such that:

$$
\begin{aligned}
& \operatorname{div} \boldsymbol{r}_{h}=-\operatorname{div}_{h} \boldsymbol{z}_{h}-u_{h}, \\
& \left\|\boldsymbol{r}_{h}\right\|+\left\|\operatorname{div} \boldsymbol{r}_{h}\right\| \lesssim\left\|-\operatorname{div}_{h} \boldsymbol{z}_{h}-u_{h}\right\|
\end{aligned}
$$

Now we define $\boldsymbol{q}_{h}=\boldsymbol{z}_{h}+\boldsymbol{r}_{h}$, noting that $\boldsymbol{r}_{h} \in H(\operatorname{div}, \Omega) \cap \boldsymbol{Q}_{h}$, namely for any $e \in \mathcal{E}_{h}^{i},\left.\left[\boldsymbol{r}_{h}\right]\right|_{e}=0$; hence, for any $e \in \mathcal{E}_{h}^{i}$ :

$$
\left.\left[\boldsymbol{q}_{h}\right]\right|_{e}=\left.\left[\boldsymbol{r}_{h}\right]\right|_{e}+\left.\left[\boldsymbol{z}_{h}\right]\right|_{e}=\left.\left[\boldsymbol{z}_{h}\right]\right|_{e}=\left.\rho h_{e} \hat{u}_{h}\right|_{e}
$$

and

$$
\operatorname{div}_{h} \boldsymbol{q}_{h}=\operatorname{div}_{h}\left(\boldsymbol{z}_{h}+\boldsymbol{r}_{h}\right)=-u_{h} .
$$

Substituting (6.32) and (6.33) into $b_{h}\left(\boldsymbol{q}_{h}, \tilde{u}_{h}\right)$, we immediately obtain:

$$
b_{h}\left(\boldsymbol{q}_{h}, \tilde{u}_{h}\right)=-\left(u_{h}, \operatorname{div}_{h} \boldsymbol{q}_{h}\right)_{\mathcal{T}_{h}}+\sum_{e \in \mathcal{E}_{h}^{i}}\left\langle\hat{u}_{h},\left[\boldsymbol{q}_{h}\right]\right\rangle_{e}=\left\|\tilde{u}_{h}\right\|_{0, \rho, h}^{2}
$$

Finally, by (6.28), (6.30), inverse inequality, and (6.29), noting that for any $e \in \mathcal{E}_{h}^{i},\left.\left[\boldsymbol{r}_{h}\right]\right|_{e}=0$, we have:

$$
\begin{aligned}
\left\|\boldsymbol{q}_{h}\right\|_{\mathrm{div}, \rho, h} & =\left\|\boldsymbol{r}_{h}+\boldsymbol{z}_{h}\right\|_{\text {Div }} \leq\left\|\boldsymbol{r}_{h}\right\|_{\text {div }}+\left\|\boldsymbol{z}_{h}\right\|_{\operatorname{div}} \leq\left\|\boldsymbol{r}_{h}\right\|+\left\|\operatorname{div} \boldsymbol{r}_{h}\right\|+\left\|\boldsymbol{z}_{h}\right\|_{\text {div }} \\
& \lesssim\left\|-\operatorname{div}_{h} \boldsymbol{z}_{h}-u_{h}\right\|+\left\|\boldsymbol{z}_{h}\right\|+\left\|\operatorname{div}_{h} \boldsymbol{z}_{h}\right\|+\left(\rho^{-1} \sum_{e} h_{e}^{-1}\left\langle\hat{P}_{e}\left(\left[\boldsymbol{z}_{h}\right]\right), \hat{P}_{e}\left(\left[\boldsymbol{z}_{h}\right]\right)\right\rangle_{e}\right)^{1 / 2} \\
& \lesssim\left\|u_{h}\right\|+\left(1+h^{-1}\right)\left\|\boldsymbol{z}_{h}\right\|+\left(\rho^{-1} \sum_{e \in \mathcal{E}_{h}^{i}} h_{e}^{-1}\left\langle\left[\boldsymbol{z}_{h}\right],\left[\boldsymbol{z}_{h}\right]\right\rangle_{e}\right)^{1 / 2} \\
& =\left\|u_{h}\right\|+\left(1+h^{-1}\right)\left(h \sum_{e \in \mathcal{E}_{h}^{i}} \rho^{2} h_{e}^{2}\left\|\hat{u}_{h}\right\|_{0, e}^{2}\right)^{1 / 2}+\left(\rho^{-1} \sum_{e \in \mathcal{E}_{h}^{i}} h_{e}^{-1}\left\langle\rho h_{e} \hat{u}_{h}, \rho h_{e} \hat{u}_{h}\right\rangle_{e}\right)^{1 / 2} \\
& \lesssim\left\|u_{h}\right\|+\left(\rho \sum_{e \in \mathcal{E}_{h}^{i}} h_{e}\left\langle\hat{u}_{h}, \hat{u}_{h}\right\rangle_{e}\right)^{1 / 2} \lesssim\left\|\tilde{u}_{h}\right\|_{0, \rho, h} .
\end{aligned}
$$

Therefore, we obtain the proof. 
Remark 6.7 In fact, we also can choose $\tau=0$ in (3.9) and for any $\tilde{v} \in \tilde{V}_{h}$, we define:

$$
\left\|\tilde{v}_{h}\right\|_{0, \rho, h}^{2}=\left(v_{h}, v_{h}\right)_{\mathcal{T}_{h}}+\sum_{e \in \mathcal{E}_{h}^{i}} h_{e}\left\langle\hat{v}_{h}, \hat{v}_{h}\right\rangle_{e}
$$

Define norms for $\boldsymbol{p}_{h} \in \boldsymbol{Q}_{h}$ as follows:

$$
\left\|\boldsymbol{p}_{h}\right\|_{\operatorname{div}, \rho, h}^{2}=\left(c \boldsymbol{p}_{h}, \boldsymbol{p}_{h}\right)_{\mathcal{T}_{h}}+\left(\operatorname{div} \boldsymbol{p}_{h}, \operatorname{div} \boldsymbol{p}_{h}\right)_{\mathcal{T}_{h}}+\sum_{e \in \mathcal{E}_{h}^{i}} h_{e}^{-1}\left\langle\hat{P}_{e}\left(\left[\boldsymbol{p}_{h}\right]\right), \hat{P}_{e}\left(\left[\boldsymbol{p}_{h}\right]\right)\right\rangle_{e}
$$

where $\hat{P}_{e}: L^{2}(e) \rightarrow \hat{V}(e)$ still is the $L^{2}$ projection. Then, we can get the stability result by a similar proof.

\subsection{Proof for Part 2 of Theorem 3.2}

Next, we prove part 2 of Theorem 3.2. The uniform boundedness of $A_{h}\left(\left(\boldsymbol{p}_{h}, \tilde{u}_{h}\right),\left(\boldsymbol{q}_{h}, \tilde{v}_{h}\right)\right)$ is obvious. The uniform inf-sup condition for $A_{h}\left(\left(\boldsymbol{p}_{h}, \tilde{u}_{h}\right),\left(\boldsymbol{q}_{h}, \tilde{v}_{h}\right)\right)$ is as follows:

Theorem 6.8 Assume $\nabla_{h} V_{h} \subset \boldsymbol{Q}_{h}$, then there exists a positive constant $\rho_{0}$ that only depends on the shape regularity of the mesh, such that for any $0<\rho \leq \rho_{0}$, we have:

$$
\inf _{\left(\boldsymbol{p}_{h}, \tilde{u}_{h}\right) \in \boldsymbol{Q}_{h} \times \tilde{V}_{h}} \sup _{\boldsymbol{q}_{h}, \tilde{v}_{h} \in \boldsymbol{Q}_{h} \times \tilde{V}_{h}} \frac{A_{h}\left(\left(\boldsymbol{p}_{h}, \tilde{u}_{h}\right),\left(\boldsymbol{q}_{h}, \tilde{v}_{h}\right)\right)}{\left(\left\|\tilde{u}_{h}\right\|_{\tilde{1}, \rho, h}+\left\|\boldsymbol{p}_{h}\right\|\right)\left(\left\|\tilde{v}_{h}\right\|_{\tilde{1}, \rho, h}+\left\|\boldsymbol{q}_{h}\right\|\right)} \geq \beta_{3},
$$

where $\beta_{3}>0$ is a constant independent of $\rho$ and mesh size $h$.

Proof. For any given $\left(\boldsymbol{p}_{h}, \tilde{u}_{h}\right) \in \boldsymbol{Q}_{h} \times \tilde{V}_{h}$, since $\nabla_{h} V_{h} \subset \boldsymbol{Q}_{h}$, we choose $\boldsymbol{q}_{h}=\boldsymbol{p}_{h}+\nabla_{h} u_{h}$ and $\tilde{v}_{h}=-\tilde{u}_{h}$, and then we have the following boundedness of $\boldsymbol{q}_{h}$ and $\tilde{v}_{h}$ by $\boldsymbol{q}_{h}$ and $\tilde{v}_{h}$

$$
\left\|\boldsymbol{q}_{h}\right\|^{2}=\left(c\left(\boldsymbol{p}_{h}+\nabla_{h} u_{h}\right), \boldsymbol{p}_{h}+\nabla_{h} u_{h}\right)_{\mathcal{T}_{h}} \leq 2\left(\left\|\boldsymbol{p}_{h}\right\|^{2}+\left\|\nabla_{h} u_{h}\right\|^{2}\right)
$$

and

$$
\left\|\tilde{v}_{h}\right\|_{\tilde{1}, \rho, h}=\left\|-\tilde{u}_{h}\right\|_{\tilde{1}, \rho, h}=\left\|\tilde{u}_{h}\right\|_{\tilde{1}, \rho, h} .
$$


On the other hand,

$$
\begin{aligned}
& A_{h}\left(\left(\boldsymbol{p}_{h}, \tilde{u}_{h}\right),\left(\boldsymbol{q}_{h}, \tilde{v}_{h}\right)\right) \\
& =\left(c \boldsymbol{p}_{h}, \boldsymbol{q}_{h}\right)_{\mathcal{T}_{h}}+\left(\nabla_{h} u_{h}, \boldsymbol{q}_{h}\right)_{\mathcal{T}_{h}}-\left\langle u_{h}-\hat{u}_{h}, \boldsymbol{q}_{h} \cdot \boldsymbol{n}\right\rangle_{\partial \mathcal{T}_{h}} \\
& +\left(\nabla_{h} v_{h}, \boldsymbol{p}_{h}\right)_{\mathcal{T}_{h}}-\left\langle v_{h}-\hat{v}_{h}, \boldsymbol{p}_{h} \cdot \boldsymbol{n}\right\rangle_{\partial \mathcal{T}_{h}}-\rho^{-1} \sum_{K \in \mathcal{T}_{h}} h_{K}^{-1}\left\langle u_{h}-\hat{u}_{h}, v_{h}-\hat{v}_{h}\right\rangle_{\partial K} \\
& =\left(c \boldsymbol{p}_{h}, \boldsymbol{p}_{h}+\nabla_{h} u_{h}\right)_{\mathcal{T}_{h}}+\left(\nabla_{h} u_{h}, \boldsymbol{p}_{h}+\nabla_{h} u_{h}\right)_{\mathcal{T}_{h}}-\left\langle u_{h}-\hat{u}_{h}, \boldsymbol{q}_{h} \cdot \boldsymbol{n}\right\rangle_{\partial \mathcal{T}_{h}} \\
& +\left(-\nabla_{h} u_{h}, \boldsymbol{p}_{h}\right)_{\mathcal{T}_{h}}-\left\langle-\left(u_{h}-\hat{u}_{h}\right), \boldsymbol{p}_{h} \cdot \boldsymbol{n}\right\rangle_{\partial \mathcal{T}_{h}}+\rho^{-1} \sum_{K \in \mathcal{T}_{h}} h_{K}^{-1}\left\langle u_{h}-\hat{u}_{h}, u_{h}-\hat{u}_{h}\right\rangle_{\partial K} \\
& =\left(c \boldsymbol{p}_{h}, \boldsymbol{p}_{h}\right)_{\mathcal{T}_{h}}+\left(c \boldsymbol{p}_{h}, \nabla_{h} u_{h}\right)_{\mathcal{T}_{h}}+\left(\nabla_{h} u_{h}, \nabla_{h} u_{h}\right)_{\mathcal{T}_{h}}+\sum_{K}\left\langle\left(u_{h}-\hat{u}_{h}\right),\left(\boldsymbol{p}_{h}-\boldsymbol{q}_{h}\right) \cdot \boldsymbol{n}_{K}\right\rangle_{\partial K} \\
& +\rho^{-1} \sum_{K \in \mathcal{T}_{h}} h_{K}^{-1}\left\langle u_{h}-\hat{u}_{h}, u_{h}-\hat{u}_{h}\right\rangle_{\partial K} \\
& \geq \frac{1}{2}\left(\left\|\boldsymbol{p}_{h}\right\|^{2}+\left\|\nabla_{h} u_{h}\right\|^{2}\right)-\epsilon C_{5}\left\|\boldsymbol{p}_{h}-\boldsymbol{q}_{h}\right\|^{2}+\left(\rho^{-1}-\epsilon^{-1}\right) \sum_{K \in \mathcal{T}_{h}} h_{K}^{-1}\left\langle u_{h}-\hat{u}_{h}, u_{h}-\hat{u}_{h}\right\rangle_{\partial K} \\
& =\frac{1}{2}\left(\left\|\boldsymbol{p}_{h}\right\|^{2}+\left\|\nabla_{h} u_{h}\right\|^{2}\right)-\epsilon C_{5}\left\|\nabla_{h} u_{h}\right\|^{2}+\left(\rho^{-1}-\epsilon^{-1}\right) \sum_{K \in \mathcal{T}_{h}} h_{K}^{-1}\left\langle u_{h}-\hat{u}_{h}, u_{h}-\hat{u}_{h}\right\rangle_{\partial K} .
\end{aligned}
$$

where $C_{5}$ is a constant independent of $\rho$ and $h$.

Now setting $\epsilon=\frac{1}{4 C_{5}}, \rho_{0}=\frac{3}{16 C_{5}}$, then for any $\rho \leq \rho_{0}$, we have:

$$
\begin{aligned}
A_{h}\left(\left(\boldsymbol{p}_{h}, \tilde{u}_{h}\right),\left(\boldsymbol{q}_{h}, \tilde{v}_{h}\right)\right) & \geq \frac{1}{2}\left\|\boldsymbol{p}_{h}\right\|^{2}+\left(\frac{1}{2}-\epsilon C_{5}\right)\left\|\nabla_{h} u_{h}\right\|^{2}+\rho^{-1}\left(1-\rho \epsilon^{-1}\right) \sum_{K \in \mathcal{T}_{h}} h_{K}^{-1}\left\langle u_{h}-\hat{u}_{h}, u_{h}-\hat{u}_{h}\right\rangle_{\partial K} \\
& \geq \frac{1}{2}\left\|\boldsymbol{p}_{h}\right\|^{2}+\frac{1}{4}\left\|\nabla_{h} u_{h}\right\|^{2}+\frac{1}{4} \rho^{-1} \sum_{K \in \mathcal{T}_{h}} h_{K}^{-1}\left\langle u_{h}-\hat{u}_{h}, u_{h}-\hat{u}_{h}\right\rangle_{\partial K} \\
& \geq \frac{1}{4}\left(\left\|\boldsymbol{p}_{h}\right\|^{2}+\left\|\tilde{u}_{h}\right\|_{\tilde{1}, \rho, h}^{2}\right) .
\end{aligned}
$$

Hereby, we complete the proof.

\subsection{Proof for Part 1 of Theorem 3.6}

By the definition of the norms, the continuity and coercivity of $a_{w}(\cdot, \cdot)$ is obvious, namely,

Theorem 6.9 For any $0<\rho \leq 1$, we have:

$$
\begin{gathered}
\left|a_{w}\left(\tilde{\boldsymbol{p}}_{h}, \tilde{\boldsymbol{q}}_{h}\right)\right| \leq\left\|\tilde{\boldsymbol{p}}_{h}\right\|_{0, h, \rho}\left\|\tilde{\boldsymbol{q}}_{h}\right\|_{0, h, \rho} \forall \quad \tilde{\boldsymbol{p}}_{h} \in \tilde{\boldsymbol{Q}}_{h}, \tilde{\boldsymbol{q}}_{h} \in \tilde{\boldsymbol{Q}}_{h} . \\
a_{w}\left(\tilde{\boldsymbol{p}}_{h}, \tilde{\boldsymbol{p}}_{h}\right) \geq\left\|\tilde{\boldsymbol{p}}_{h}\right\|_{0, h, \rho}^{2} \forall \tilde{\boldsymbol{p}}_{h} \in \tilde{\boldsymbol{Q}}_{h} .
\end{gathered}
$$

Before we prove the boundedness and inf-sup condition of $b_{w}\left(\tilde{\boldsymbol{p}}_{h}, v_{h}\right)$, by identity $(2.2)$ and noting that $\left[\hat{\boldsymbol{p}}_{h}\right]=0$, we rewrite $b_{w}\left(\tilde{\boldsymbol{p}}_{h}, v_{h}\right)$ as:

$$
b_{w}\left(\tilde{\boldsymbol{p}}_{h}, v_{h}\right)=\left(\boldsymbol{p}_{h}, \nabla_{h} v_{h}\right)_{\mathcal{T}_{h}}-\left(\hat{\boldsymbol{p}}_{h} \cdot \boldsymbol{n}_{K}, v_{h}\right)_{\partial \mathcal{T}_{h}}=\left(\boldsymbol{p}_{h}, \nabla v_{h}\right)_{\mathcal{T}_{h}}-\left\langle\hat{\boldsymbol{p}}_{h}, \llbracket v_{h} \rrbracket\right\rangle_{\mathcal{E}_{h}} .
$$


Then, the boundedness of $b_{w}\left(\tilde{\boldsymbol{p}}_{h}, v_{h}\right)$ is as follows:

Theorem 6.10 For any $0<\rho \leq 1$, and for any $\tilde{\boldsymbol{p}}_{h} \in \tilde{\boldsymbol{Q}}_{h}, v_{h} \in V_{h}$,

$$
b_{w}\left(\tilde{\boldsymbol{p}}_{h}, v_{h}\right) \leq C_{w}\left\|\tilde{\boldsymbol{p}}_{h}\right\|_{0, h \rho}\left\|v_{h}\right\|_{1, h, \rho}
$$

Proof. Using the Cauchy inequality for (6.41), we obtain:

$$
\begin{aligned}
\left|b_{w}\left(\tilde{\boldsymbol{p}}_{h}, v_{h}\right)\right| & \leq\left\|\boldsymbol{p}_{h}\right\|\left\|\nabla_{h} v_{h}\right\|+\sum_{e \in \mathcal{E}_{h}}\left\|\hat{\boldsymbol{p}}_{h} \cdot \boldsymbol{n}_{e}\right\|_{0, e}\left\|\hat{Q}_{e}\left(\left[v_{h}\right]\right)\right\|_{0, e} \\
& \leq\left\|\boldsymbol{p}_{h}\right\|\left\|\nabla_{h} v_{h}\right\|+\left(\rho \sum_{e \in \mathcal{E}_{h}} h_{e}\left\|\hat{\boldsymbol{p}}_{h} \cdot \boldsymbol{n}_{e}\right\|_{0, e}^{2}\right)^{1 / 2}\left(\rho^{-1} \sum_{e \in \mathcal{E}_{h}} h_{e}^{-1}\left\|\hat{Q}_{e}\left(\left[v_{h}\right]\right)\right\|_{0, e}^{2}\right)^{1 / 2} \\
& \leq\left(\left\|\boldsymbol{p}_{h}\right\|+\left(\rho \sum_{e \in \mathcal{E}_{h}} h_{e}\left\|\hat{\boldsymbol{p}}_{h} \cdot \boldsymbol{n}_{e}\right\|_{0, e}^{2}\right)^{1 / 2}\right)\left\|v_{h}\right\|_{1, h, \rho} .
\end{aligned}
$$

Let $K$ be an element that takes e as an edge or flat face. Then, using the trace inequality and the inverse inequality we obtain:

$$
h_{e}\left\|\hat{\boldsymbol{p}}_{h} \cdot \boldsymbol{n}_{e}\right\|_{0, e}^{2} \leq 2 h_{e}\left\|\left(\hat{\boldsymbol{p}}_{h}-\boldsymbol{p}_{h}\right) \cdot \boldsymbol{n}_{e}\right\|_{0, e}^{2}+2 h_{e}\left\|\boldsymbol{p}_{h} \cdot \boldsymbol{n}_{e}\right\|_{0, e}^{2} \leq C_{t}\left(h_{e}\left\|\left(\hat{\boldsymbol{p}}_{h}-\boldsymbol{p}_{h}\right) \cdot \boldsymbol{n}_{e}\right\|_{0, e}^{2}+\left\|\boldsymbol{p}_{h}\right\|_{0, K}^{2}\right)
$$

Substituting the above inequality (6.44) into (6.43) yields:

$$
b_{w}\left(\tilde{\boldsymbol{p}}_{h}, v_{h}\right) \leq C_{w}\left\|\tilde{\boldsymbol{p}}_{h}\right\|_{0, h, \rho}\left\|v_{h}\right\|_{1, h, \rho}
$$

Hence, the lemma is proved.

We also have the following uniform inf-sup condition for $b_{w}\left(\tilde{\boldsymbol{p}}_{h}, v_{h}\right)$ :

Theorem 6.11 Assume $\nabla_{h} V_{h} \subset \boldsymbol{Q}_{h}$, then for any $0<\rho \leq 1$, we have:

$$
\inf _{v_{h} \in V_{h}} \sup _{\tilde{\boldsymbol{p}}_{h} \in \tilde{\boldsymbol{Q}}_{h}} \frac{b_{w}\left(\tilde{\boldsymbol{p}}_{h}, v_{h}\right)}{\left\|v_{h}\right\|_{1, h, \rho}\left\|\tilde{\boldsymbol{p}}_{h}\right\|_{0, h, \rho}} \geq \beta_{4}
$$

where $\beta_{4}>0$ is independent of mesh size $h$ and $\rho$.

Proof. Since $\nabla_{h} V_{h} \subset \boldsymbol{Q}_{h}$, taking $\boldsymbol{p}_{h}=\nabla_{h} v_{h}, \hat{\boldsymbol{p}}_{h}=-\rho^{-1} h_{e}^{-1} \hat{Q}_{e}\left(\left[v_{h}\right]\right) \boldsymbol{n}_{e}$ in $(6.41)$, we have

$$
b_{w}\left(\tilde{\boldsymbol{p}}_{h}, v_{h}\right)=\left(\nabla_{h} v_{h}, \nabla_{h} v_{h}\right)_{\mathcal{T}_{h}}+\rho^{-1} \sum_{e \in \mathcal{E}_{h}} h_{e}^{-1}\left\langle\hat{Q}_{e}\left(\left[v_{h}\right]\right), \hat{Q}_{e}\left(\left[v_{h}\right]\right)\right\rangle_{e}=\left\|v_{h}\right\|_{1, h, \rho}^{2}
$$

Noting that $\rho \leq 1$, we obtain:

$$
\begin{aligned}
\left\|\tilde{\boldsymbol{p}}_{h}\right\|_{0, h, \rho}^{2} & =\left(c \nabla_{h} v_{h}, \nabla_{h} v_{h}\right)_{\mathcal{T}_{h}}+\rho \sum_{K \in \mathcal{T}_{h}} h_{K}\left\|\nabla_{h} v_{h} \cdot \boldsymbol{n}_{\boldsymbol{K}}+\rho^{-1} h_{e}^{-1} \hat{Q}_{e}\left(\left[v_{h}\right]\right) \boldsymbol{n}_{e} \cdot \boldsymbol{n}_{K}\right\|_{0, \partial K}^{2} \\
& \leq \beta_{4}\left(\left(c \nabla_{h} v_{h}, \nabla_{h} v_{h}\right)_{\mathcal{T}_{h}}+\rho^{-1} \sum_{e \in \mathcal{E}_{h}} h_{e}^{-1}\left\|\hat{Q}_{e}\left(\left[v_{h}\right]\right)\right\|_{0, e}^{2}\right) \\
& \leq \beta_{4}\left\|v_{h}\right\|_{1, h, \rho}^{2} .
\end{aligned}
$$

Here, we obtain the desired result. 


\subsection{Proof for Part 2 of Theorem 3.6}

Next, we prove part 2 of Theorem 2. The uniform boundedness of $A_{w}((\cdot, \cdot),(\cdot, \cdot))$ is obvious. The uniform inf-sup of $A_{w}((\cdot, \cdot),(\cdot, \cdot))$ is as follows:

Theorem 6.12 Let $\boldsymbol{R}_{h} \subset H(\operatorname{div}, \Omega) \cap \boldsymbol{Q}_{h}$ be the Raviart-Thomas finite element space. Assume that $\left\{\left\{\boldsymbol{R}_{h}\right\}\right\} \subset$ $\hat{Q}_{h}$ and $V_{h}=\operatorname{div}_{h} \boldsymbol{Q}_{h}$. Then, for $0<\rho \leq 1$ the bilinear form $A_{w}((\cdot, \cdot),(\cdot, \cdot))$ with $\eta=\rho^{-1} h_{K}^{-1}$ satisfies:

$$
\inf _{\left(\tilde{\boldsymbol{p}}_{h}, u_{h}\right) \in \tilde{\boldsymbol{Q}}_{h} \times V_{h}} \sup _{\left(\tilde{\boldsymbol{q}}_{h}, v_{h}\right) \in \tilde{\boldsymbol{Q}}_{h} \times V_{h}} \frac{A_{w}\left(\left(\tilde{\boldsymbol{p}}_{h}, u_{h}\right),\left(\tilde{\boldsymbol{q}}_{h}, v_{h}\right)\right)}{\left(\left\|u_{h}\right\|+\left\|\tilde{\boldsymbol{p}}_{h}\right\|_{\widetilde{\mathrm{div}}, \rho, h}\right)\left(\left\|v_{h}\right\|+\left\|\tilde{\boldsymbol{q}}_{h}\right\|_{\widetilde{\mathrm{div}}, \rho, h}\right)} \geq \beta_{5},
$$

where $\beta_{5}>0$ is a constant independent of both $\rho$ and mesh size $h$.

Proof. For any given $\left(\tilde{\boldsymbol{p}}_{h}, u_{h}\right) \in \tilde{\boldsymbol{Q}}_{h} \times V_{h}$, namely $\left(\boldsymbol{p}_{h}, \hat{\boldsymbol{p}}_{h}, u_{h}\right) \in \boldsymbol{Q}_{h} \times \hat{Q}_{h} \times V_{h}$. Since $V_{h} \subset \operatorname{div} \boldsymbol{R}_{h}$ and $\boldsymbol{R}_{h} \times V_{h}$ such that the mixed conforming method is well-defined, there exists $\boldsymbol{r}_{h} \in \boldsymbol{R}_{h}$ such that:

$$
-\operatorname{div} \boldsymbol{r}_{h}=u_{h} \text { and }\left\|\boldsymbol{r}_{h}\right\|+\left\|\operatorname{div} \boldsymbol{r}_{h}\right\| \leq C\left\|u_{h}\right\|
$$

Now we choose $\boldsymbol{q}_{h}=\boldsymbol{r}_{h}+\alpha \boldsymbol{p}_{h}, \hat{\boldsymbol{q}}_{h}=\alpha \hat{\boldsymbol{p}}_{h}+\left(\boldsymbol{r}_{h} \cdot \boldsymbol{n}_{e}\right) \boldsymbol{n}_{e}, v_{h}=-\operatorname{div}_{h} \boldsymbol{p}_{h}-\alpha u_{h}$, where $\alpha$ is a constant that will be indicated later.

We then first verify the boundedness of $\left(\tilde{\boldsymbol{q}}_{h}, v_{h}\right)$ by $\left(\tilde{\boldsymbol{p}}_{h}, u_{h}\right)$.

Noting that $\left.\left(\boldsymbol{q}_{h}-\hat{\boldsymbol{q}}_{h}\right) \cdot \boldsymbol{n}_{K}\right|_{\partial K}=\left.\left(\boldsymbol{r}_{h}+\alpha \boldsymbol{p}_{h}-\alpha \hat{\boldsymbol{p}}_{h}-\left(\boldsymbol{r}_{h} \cdot \boldsymbol{n}_{e}\right) \boldsymbol{n}_{e}\right) \cdot \boldsymbol{n}_{K}\right|_{\partial K}=\left.\alpha\left(\boldsymbol{p}_{h}-\hat{\boldsymbol{p}}_{h}\right) \cdot \boldsymbol{n}_{K}\right|_{\partial K}$, we have:

$$
\begin{aligned}
\left\|\tilde{\boldsymbol{q}}_{h}\right\|_{\widetilde{\operatorname{div}}, \rho, K}^{2} & =\left(c \boldsymbol{q}_{h}, \boldsymbol{q}_{h}\right)_{K}+\left(\operatorname{div} \boldsymbol{q}_{h}, \operatorname{div} \boldsymbol{q}_{h}\right)_{K}+\rho^{-1} h_{K}^{-1}\left\langle\left(\boldsymbol{q}_{h}-\hat{\boldsymbol{q}}_{h}\right) \cdot \boldsymbol{n}_{K},\left(\boldsymbol{q}_{h}-\hat{\boldsymbol{q}}_{h}\right) \cdot \boldsymbol{n}_{K}\right\rangle_{\partial K} \\
& =\left(c\left(\boldsymbol{r}_{h}+\alpha \boldsymbol{p}_{h}\right), \boldsymbol{r}_{h}+\alpha \boldsymbol{p}_{h}\right)_{K}+\left(\operatorname{div} \boldsymbol{r}_{h}+\alpha \operatorname{div} \boldsymbol{p}_{h}, \operatorname{div} \boldsymbol{r}_{h}+\alpha \operatorname{div} \boldsymbol{p}_{h}\right)_{K} \\
& +\alpha^{2} \rho^{-1} h_{K}^{-1}\left\langle\left(\boldsymbol{p}_{h}-\hat{\boldsymbol{p}}_{h}\right) \cdot \boldsymbol{n}_{K},\left(\boldsymbol{p}_{h}-\hat{\boldsymbol{p}}_{h}\right) \cdot \boldsymbol{n}_{K}\right\rangle_{\partial K} \\
& \leq 2\left\|\boldsymbol{r}_{h}\right\|^{2}+2 \alpha^{2}\left\|\boldsymbol{p}_{h}\right\|^{2}+2\left\|\operatorname{div} \boldsymbol{r}_{h}\right\|^{2}+\left(\operatorname{div}_{h} \boldsymbol{p}_{h}, \operatorname{div}_{h} \boldsymbol{p}_{h}\right) \\
& +\alpha^{2} \rho^{-1} h_{K}^{-1}\left\langle\left(\boldsymbol{p}_{h}-\hat{\boldsymbol{p}}_{h}\right) \cdot \boldsymbol{n}_{K},\left(\boldsymbol{p}_{h}-\hat{\boldsymbol{p}}_{h}\right) \cdot \boldsymbol{n}_{K}\right\rangle_{\partial K} \\
& \leq 2\left(C^{2}\left\|u_{h}\right\|_{0, K}^{2}+\alpha^{2}\left\|\tilde{\boldsymbol{p}}_{h}\right\|_{\widetilde{\mathrm{div}}, \rho, K}^{2}\right) .
\end{aligned}
$$

Hence, $\left\|\tilde{\boldsymbol{q}}_{h}\right\|_{\widetilde{\mathrm{div}}, h, \rho}^{2} \leq 2\left(C^{2}\left\|u_{h}\right\|^{2}+\alpha^{2}\left\|\tilde{\boldsymbol{p}}_{h}\right\|_{\text {div }, h, \rho}^{2}\right)$. Further,

$$
\left\|v_{h}\right\|=\left\|-\operatorname{div}_{h} \boldsymbol{p}_{h}-\alpha u_{h}\right\|=\left\|\operatorname{div}_{h} \boldsymbol{p}_{h}\right\|+\alpha\left\|u_{h}\right\| \leq\left\|\tilde{\boldsymbol{p}}_{h}\right\|_{\widetilde{\mathrm{div}}, h, \rho}+\alpha\left\|u_{h}\right\| .
$$

Then, we prove the boundedness of $\left(\tilde{\boldsymbol{q}}_{h}, v_{h}\right)$ by $\left(\tilde{\boldsymbol{p}}_{h}, u_{h}\right)$. 
Now through integration by parts, we have the following:

$$
\begin{aligned}
& A_{w}\left(\left(\tilde{\boldsymbol{p}}_{h}, u_{h}\right),\left(\tilde{\boldsymbol{q}}_{h}, v_{h}\right)\right) \\
= & \left(c \boldsymbol{p}_{h}, \boldsymbol{q}_{h}\right)_{\mathcal{T}_{h}}+\rho^{-1} \sum_{K \in \mathcal{T}_{h}} h_{K}^{-1}\left\langle\left(\boldsymbol{p}_{h}-\hat{\boldsymbol{p}}_{h}\right) \cdot \boldsymbol{n}_{K},\left(\boldsymbol{q}_{h}-\hat{\boldsymbol{q}}_{h}\right) \cdot \boldsymbol{n}_{K}\right\rangle_{\partial K} \\
& +\left(\boldsymbol{q}_{h}, \nabla_{h} u_{h}\right)_{\mathcal{T}_{h}}-\left\langle\hat{\boldsymbol{q}}_{h} \cdot \boldsymbol{n}, u_{h}\right\rangle_{\partial \mathcal{T}_{h}}+\left(\boldsymbol{p}_{h}, \nabla_{h} v_{h}\right)_{\mathcal{T}_{h}}-\left\langle\hat{\boldsymbol{p}}_{h} \cdot \boldsymbol{n}, v_{h}\right\rangle_{\partial \mathcal{T}_{h}} \\
= & \left(c \boldsymbol{p}_{h}, \boldsymbol{q}_{h}\right)_{\mathcal{T}_{h}}+\rho^{-1} \sum_{K \in \mathcal{T}_{h}} h_{K}^{-1}\left\langle\left(\boldsymbol{p}_{h}-\hat{\boldsymbol{p}}_{h}\right) \cdot \boldsymbol{n}_{K},\left(\boldsymbol{q}_{h}-\hat{\boldsymbol{q}}_{h}\right) \cdot \boldsymbol{n}_{K}\right\rangle_{\partial K}-\left(\operatorname{div}_{h} \boldsymbol{q}_{h}, u_{h}\right)_{\mathcal{T}_{h}} \\
& +\left\langle\left(\boldsymbol{q}_{h}-\hat{\boldsymbol{q}}_{h}\right) \cdot \boldsymbol{n}, u_{h}\right\rangle_{\partial \mathcal{T}_{h}}-\left(\operatorname{div}_{h} \boldsymbol{p}_{h}, v_{h}\right)_{\mathcal{T}_{h}}+\left\langle\left(\boldsymbol{p}_{h}-\hat{\boldsymbol{p}}_{h}\right) \cdot \boldsymbol{n}, v_{h}\right\rangle_{\partial \mathcal{T}_{h}}
\end{aligned}
$$

By the Cauchy inequality and inverse inequality, we have:

$$
\begin{aligned}
A_{w, K} & \left(\left(\tilde{\boldsymbol{p}}_{h}, u_{h}\right),\left(\tilde{\boldsymbol{q}}_{h}, v_{h}\right)\right) \\
= & \left(c \boldsymbol{p}_{h}, \boldsymbol{r}_{h}+\alpha \boldsymbol{p}_{h}\right)_{K}+\alpha \rho^{-1} h_{K}^{-1}\left\langle\left(\boldsymbol{p}_{h}-\hat{\boldsymbol{p}}_{h}\right) \cdot \boldsymbol{n}_{K},\left(\boldsymbol{p}_{h}-\hat{\boldsymbol{p}}_{h}\right) \cdot \boldsymbol{n}_{K}\right\rangle_{\partial K} \\
& -\left(\operatorname{div} \boldsymbol{r}_{h}+\alpha \operatorname{div} \boldsymbol{p}_{h}, u_{h}\right)_{K}+\alpha\left\langle\left(\boldsymbol{p}_{h}-\hat{\boldsymbol{p}}_{h}\right) \cdot \boldsymbol{n}_{K}, u_{h}\right\rangle_{\partial K} \\
& -\left(\operatorname{div} \boldsymbol{p}_{h},-\operatorname{div} \boldsymbol{p}_{h}-\alpha u_{h}\right)_{K}+\left\langle\left(\boldsymbol{p}_{h}-\hat{\boldsymbol{p}}_{h}\right) \cdot \boldsymbol{n}_{K},-\operatorname{div} \boldsymbol{p}_{h}-\alpha u_{h}\right\rangle_{\partial K} \\
= & \left(c \boldsymbol{p}_{h}, \boldsymbol{r}_{h}\right)_{K}+\alpha\left(c \boldsymbol{p}_{h}, \boldsymbol{p}_{h}\right)_{K}+\alpha \rho^{-1} h_{K}^{-1}\left\langle\left(\boldsymbol{p}_{h}-\hat{\boldsymbol{p}}_{h}\right) \cdot \boldsymbol{n}_{K},\left(\boldsymbol{p}_{h}-\hat{\boldsymbol{p}}_{h}\right) \cdot \boldsymbol{n}_{K}\right\rangle_{\partial K} \\
& +\left(u_{h}, u_{h}\right)_{K}+\left(\operatorname{div} \boldsymbol{p}_{h}, \operatorname{div} \boldsymbol{p}_{h}\right)_{K}-\left\langle\left(\boldsymbol{p}_{h}-\hat{\boldsymbol{p}}_{h}\right) \cdot \boldsymbol{n}_{K}, \operatorname{div} \boldsymbol{p}_{h}\right\rangle_{\partial K} \\
\geq & -\epsilon_{1}\left\|\boldsymbol{r}_{h}\right\|_{0, K}^{2}-\epsilon_{1}^{-1}\left\|\boldsymbol{p}_{h}\right\|_{0, K}^{2}+\alpha\left\|\boldsymbol{p}_{h}\right\|_{0, K}^{2}+\alpha \rho^{-1} h_{K}^{-1}\left\langle\left(\boldsymbol{p}_{h}-\hat{\boldsymbol{p}}_{h}\right) \cdot \boldsymbol{n}_{K},\left(\boldsymbol{p}_{h}-\hat{\boldsymbol{p}}_{h}\right) \cdot \boldsymbol{n}_{K}\right\rangle_{\partial K} \\
& +\left\|u_{h}\right\|_{0, K}^{2}+\left(\operatorname{div} \boldsymbol{p}_{h}, \operatorname{div} \boldsymbol{p}_{h}\right)_{K}-\epsilon_{2}^{-1} h_{K}^{-1}\left\langle\left(\boldsymbol{p}_{h}-\hat{\boldsymbol{p}}_{h}\right) \cdot \boldsymbol{n}_{K},\left(\boldsymbol{p}_{h}-\hat{\boldsymbol{p}}_{h}\right) \cdot \boldsymbol{n}_{K}\right\rangle_{\partial K} \\
& -\epsilon_{2} h_{K}\left(\operatorname{div} \boldsymbol{p}_{h}, \operatorname{div} \boldsymbol{p}_{h}\right)_{\partial K} \\
= & -\epsilon_{1}\left\|\boldsymbol{r}_{h}\right\|_{0, K}^{2}+\left(\alpha-\epsilon_{1}^{-1}\right)\left\|\boldsymbol{p}_{h}\right\|_{0, K}^{2}+\left\|u_{h}\right\|_{0, K}^{2}+\left(1-\epsilon_{2} C_{6}\right)\left(\operatorname{div} \boldsymbol{p}_{h}, \operatorname{div} \boldsymbol{p}_{h}\right)_{K} \\
& +\left(\alpha \rho^{-1}-\epsilon_{2}^{-1}\right) h_{K}^{-1}\left\langle\left(\boldsymbol{p}_{h}-\hat{\boldsymbol{p}}_{h}\right) \cdot \boldsymbol{n}_{K},\left(\boldsymbol{p}_{h}-\hat{\boldsymbol{p}}_{h}\right) \cdot \boldsymbol{n}_{K}\right\rangle_{\partial K}
\end{aligned}
$$

Noting (6.48), we have the following:

$$
\begin{aligned}
A_{w}\left(\left(\tilde{\boldsymbol{p}}_{h}, u_{h}\right),\left(\tilde{\boldsymbol{q}}_{h}, v_{h}\right)\right) \geq & \left(1-\epsilon_{1} C\right)\left\|u_{h}\right\|^{2}+\left(\alpha-\epsilon_{1}^{-1}\right)\left\|\boldsymbol{p}_{h}\right\|^{2}+\left(1-\epsilon_{2} C_{6}\right)\left(\operatorname{div}_{h} \boldsymbol{p}_{h}, \operatorname{div}_{h} \boldsymbol{p}_{h}\right) \\
& +\left(\alpha \rho^{-1}-\epsilon_{2}^{-1}\right) \sum_{K \in \mathcal{T}_{h}} h_{K}^{-1}\left\langle\left(\boldsymbol{p}_{h}-\hat{\boldsymbol{p}}_{h}\right) \cdot \boldsymbol{n}_{K},\left(\boldsymbol{p}_{h}-\hat{\boldsymbol{p}}_{h}\right) \cdot \boldsymbol{n}_{K}\right\rangle_{\partial K} .
\end{aligned}
$$

Now choosing $\epsilon_{1}=\frac{1}{2 C}, \epsilon_{2}=\frac{1}{2 C_{6}}, \alpha=\max \left\{2 C+\frac{1}{2}, 2 C_{6}+\frac{1}{2}\right\}, 0<\rho \leq 1$, we have:

$$
\begin{aligned}
A_{w}\left(\left(\tilde{\boldsymbol{p}}_{h}, u_{h}\right),\left(\tilde{\boldsymbol{q}}_{h}, v_{h}\right)\right) & \geq \frac{1}{2}\left(\left\|\boldsymbol{p}_{h}\right\|^{2}+\sum_{K \in \mathcal{T}_{h}} h_{K}^{-1}\left\|\left(\boldsymbol{p}_{h}-\hat{\boldsymbol{p}}_{h}\right) \cdot \boldsymbol{n}_{K}\right\|_{0, \partial K}^{2}+\left\|u_{h}\right\|^{2}+\left\|\operatorname{div}_{h} \boldsymbol{p}_{h}\right\|^{2}\right) \\
& \geq \frac{1}{2}\left(\left\|u_{h}\right\|^{2}+\left\|\tilde{\boldsymbol{p}}_{h}\right\|_{\widetilde{\mathrm{div}}, h, \rho}\right) .
\end{aligned}
$$

Thus, we prove the theorem. 


\section{$7 \quad$ Summary}

In this paper we use the classic LBB theory to prove two types of uniform stability results under some proper parameter-dependent norms for HDG methods, which are uniformly stable with respect to the stabilization parameters and mesh size $h$. Based on the uniform stability results, we further prove uniform and optimal error estimates for HDG methods, which are independent of the stabilization parameters. In addition, we also prove two types of uniform stability results for WG methods. Similarly based on the uniform stability results, we further prove uniform and optimal error estimates for WG methods. These uniform stability results and optimal error estimates for WG methods are meaningful. Following these uniform stability results for HDG methods and WG methods presented in this paper, an HDG method is shown to converge to a primal conforming method, whereas a WG method is shown to converge to a mixed conforming method by taking the limit of the stabilization parameters.

\section{References}

[1] George Em Karniadakis, Chi-Wang Shu, and Bernardo Cockburn. Discontinuous Galerkin Methods: Theory, Computation and Applications. Springer, 2000.

[2] Douglas N Arnold, Franco Brezzi, Bernardo Cockburn, and L Donatella Marini. Unified analysis of discontinuous Galerkin methods for elliptic problems. SIAM Journal on Numerical Analysis, 39(5):1749$1779,2002$.

[3] BX Fraeijs de Veubeke. Displacement and equilibrium models in the finite element method. Stress analysis, pages 275-284, 1965.

[4] Bernardo Cockburn and Jayadeep Gopalakrishnan. A characterization of hybridized mixed methods for second order elliptic problems. SIAM Journal on Numerical Analysis, 42(1):283-301, 2004.

[5] Jesús Carrero, Bernardo Cockburn, and Dominik Schötzau. Hybridized globally divergence-free LDG methods. Part I: The Stokes problem. Mathematics of Computation, 75(254):533-563, 2006.

[6] Bo Dong Cockburn, Bernardo and Johnny Guzmn. A superconvergent LDG-hybridizable Galerkin method for second-order elliptic problems. Mathematics of Computation, 77(264):1887-1916, 2008.

[7] Bernardo Cockburn, Jayadeep Gopalakrishnan, and Raytcho Lazarov. Unified hybridization of discontinuous Galerkin, mixed, and continuous Galerkin methods for second order elliptic problems. SIAM Journal on Numerical Analysis, 47(2):1319-1365, 2009. 
[8] Jayadeep Gopalakrishnan Cockburn, Bernardo and Francisco-Javier Sayas. A projection-based error analysis of HDG methods. Mathematics of Computation, 79(271):1351-1367, 2010.

[9] Yanlai Chen and Bernardo Cockburn. Analysis of variable-degree hdg methods for convection-diffusion equations. part ii: Semimatching nonconforming meshes. Mathematics of Computation, 83(285):87-111, 2014.

[10] Issei Oikawa. A hybridized discontinuous galerkin method with reduced stabilization. Journal of Scientific Computing, 65(1):327-340, 2015.

[11] Junping Wang and Xiu Ye. A weak Galerkin finite element method for second-order elliptic problems. Journal of Computational and Applied Mathematics, 241:103-115, 2013.

[12] Junping Wang and Xiu Ye. A weak Galerkin mixed finite element method for second order elliptic problems. Mathematics of Computation, 83(289):2101-2126, 2014.

[13] Wenbin Chen, Fang Wang, and Yanqiu Wang. Weak galerkin method for the coupled darcy-stokes flow. IMA Journal of Numerical Analysis, 36(2):897-921, 2016.

[14] Chunmei Wang and Junping Wang. A primal-dual weak galerkin finite element method for second order elliptic equations in non-divergence form. Mathematics of Computation, 2017.

[15] Chunmei Wang and Junping Wang. A primal-dual weak galerkin finite element method for fokker-planck type equations. arXiv preprint arXiv:1704.05606, 2017.

[16] Bernardo Cockburn. Static condensation, hybridization, and the devising of the HDG methods. In Building Bridges: Connections and Challenges in Modern Approaches to Numerical Partial Differential Equations, pages 129-177. Springer, 2016.

[17] Junping Wang and Chunmei Wang. Weak galerkin finite element methods for elliptic pdes. SCIENTIA SINICA Mathematica, 45(7):1061-1092, 2015.

[18] Philippe G Ciarlet. The finite element method for elliptic problems, volume 4 of Studies in Mathematics and its Applications. North-Holland, 1978.

[19] S.C. Brenner. Poincaré-friedrichs inequalities for piecewise h 1 functions. SIAM Journal on Numerical Analysis, 41(1):306-324, 2003.

[20] Hong Qingguo, Wang Fei, Wu Shuonan, and Xu Jinchao. A unified study of continuous and discontinuous galerkin methods. SCIENCE CHINA Mathematics. 
[21] Susanne Brenner and Ridgway Scott. The mathematical theory of finite element methods, volume 15. Springer Science \& Business Media, 2007.

[22] Susanne C Brenner and Li-Yeng Sung. C 0 interior penalty methods for fourth order elliptic boundary value problems on polygonal domains. Journal of Scientific Computing, 22(1-3):83-118, 2005.

[23] Franco Brezzi and Michel Fortin. Mixed and hybrid finite element methods, volume 15 of Springer Series in Computational Mathematics. Springer-Verlag, 1991.

[24] Daniele Boffi, Franco Brezzi, and Michel Fortin. Mixed finite element methods and applications, volume 44 of Springer Series in Computational Mathematics. Springer, 2013. 